\title{
The necessity and sufficiency of anytime capacity for control over a noisy communication link: Parts I and II
}

\author{
Anant Sahai; Sanjoy Mitter ${ }^{\dagger}$ \\ sahai@eecs.berkeley.edu, mitter@mit.edu
}

\begin{abstract}
We review how Shannon's classical notion of capacity is not enough to characterize a noisy communication channel if we intend to use that channel as a part of a feedback loop to stabilize an unstable linear system. While classical capacity is not enough, another parametric sense of capacity called "anytime capacity" is shown to be necessary for the stabilization of an unstable process. The rate required is given by the log of the system gain and the sense of reliability required comes from the sense of stability desired. A consequence of this necessity result is a sequential generalization of the Schalkwijk/Kailath scheme for communication over the AWGN channel with feedback.

In cases of sufficiently rich information patterns between the encoder and decoder, we show that adequate anytime capacity is also sufficient for there to exist a stabilizing controller. These sufficiency results are then generalized to cases with noisy observations and without any explicit feedback between the observer and the controller. Both necessary and sufficient conditions are extended to the continuous time case as well.

In part II, the vector-state generalizations are established. Here, it is the magnitudes of the unstable eigenvalues that play an essential role. The channel is no longer summarized by a single number, or even a single function. Instead, the concept of the anytime-rate-region is introduced in which we ask for the region of rates that the channel can support while still meeting different reliability targets for parallel bitstreams. For cases where there is no explicit feedback of the noisy channel outputs, the intrinsic delay of the control system tells us what the feedback delay needs to be while evaluating the anytime reliability of the channel.
\end{abstract}

\section{Introduction}

For communication theorists, Shannon's classical channel capacity theorems are not just beautiful mathematical results, they are useful in practice as well. They let us summarize a diverse range of channels by a single figure of merit: the capacity. For most non-interactive point-to-point communication applications, the Shannon capacity of a channel provides an upper bound on performance in

* A. Sahai is with the Department of Electrical Engineering and Computer Science, U.C. Berkeley. Early portions of this work appeared in his doctoral dissertation and a few other results will be presented at the 2004 Conference on Decision and Control.

${ }^{\dagger}$ Department of Electrical Engineering and Computer Science at the Massachusetts Institute of Technology. 
terms of end-to-end distortion through the distortion-rate function. As far as distortion is concerned, all that matters is the channel capacity and the nature of the source. At the same time, the coding theorems tell us that given enough of a tolerance for end-to-end delay, the source can be encoded into bits and those bits can be reliably transported across the noisy channel if the rate is less than the Shannon capacity. As long as the source, distortion, and channel are well-behaved[9, 37], there is asymptotically no loss in separating the problem of source and channel coding. This provides us with a justification for a layered architecture that lets engineers isolate the problem of reliable communication from that of using the communicated information. After five decades, recent advances in computational tools and coding theory have also made it possible to approach the capacity bounds very closely in practical systems.

In order to extend our understanding of communication to interactive settings, it is essential to have some model for interaction. Schulman and others have studied interaction in the context of distributed computation.[26, 16] The interaction there was between computational agents that each had access to some private data and wished to perform a global computation in a distributed way. The computational agents could only communicate with each other through noisy channels. Capacity was not a question of major interest since polynomial or constant factor slowdowns were considered acceptable. Fundamentally, this was a consequence of being able to design all the system dynamics. The rich field of automatic control provides an interactive context to study capacity requirements since the plant dynamics are considered as a given, rather than something that can be designed. In control, we will consider interaction between an observer that gets to see the plant and a controller that gets to control it. These two will be connected by a noisy channel.

Shannon himself had suggested looking to control problems for more insight into reliable communication. [29]

"... can be pursued further and is related to a duality between past and future and the notions of control and knowledge. Thus we may have knowledge of the past and cannot control it; we may control the future but have no knowledge of it."

It is therefore natural to ask whether Shannon's classical capacity is the appropriate characterization for communication channels being used in distributed control systems. Recent work on sequential rate distortion theory has shown that there is a fundamental rate of information production, namely the sum of the logs of the unstable eigenvalues of the plant, that is invariantly attached to an unstable linear discrete-time process. [34, 35] This particular notion of rate was justified by showing how to stabilize the system over a noiseless digital feedback link with any capacity greater than the intrinsic rate for the unstable process, as well as showing that it is impossible to stabilize the system using a noiseless channel of lower capacity. In addition to noiseless channels, the results were extended for almost-sure stabilization in the context of undisturbed ${ }^{1}$ control systems with unknown but bounded initial conditions being stabilized over noisy channels.[36]

We had previously showed that it is possible to stabilize persistently disturbed controlled GaussMarkov processes over suitable noisy continuous valued power-constrained AWGN (Additive White Gaussian Noise) channels[17, 33] where it turned out that Shannon capacity was tight and linear observers and controllers were sufficient.[1] Next, we had studied the question of stabilization in the context of a discrete channel: the binary erasure channel. There we showed that Shannon capacity is not sufficient for capacity and introduced the anytime capacity as a candidate figure of merit.[18] Subsequently, there was also related work by Elia that used ideas from robust control to deal with communication uncertainty in a mixed continuous/discrete context.[7,8] The area of control with

\footnotetext{
${ }^{1}$ In seminal work [36], there is no persistent disturbance acting on the unstable plant.
} 


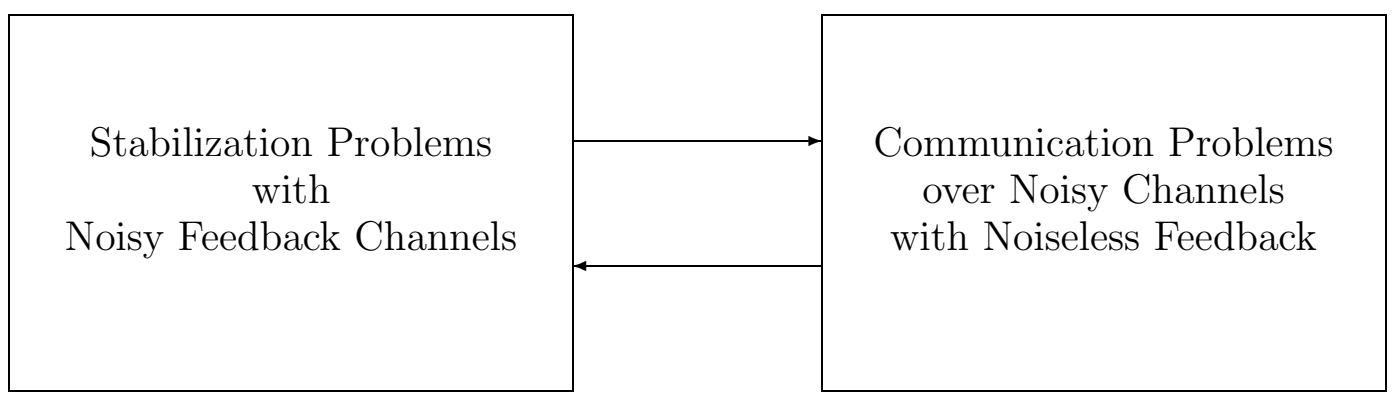

Figure 1: The equivalence between stabilization over noisy feedback channels and reliable communication over noisy channels with feedback is the main result established in this paper.

communications constraints continues to attract attention and the reader is directed to the recent September 2004 issue of IEEE Transactions on Automatic Control and the articles therein for a more comprehensive survey.

Many of the issues that arise in the context of control here in this paper also arise for the conceptually simpler problem of merely estimating an unstable open-loop process over a noisy channel. For this estimation problem in the limit of large, but finite, end-to-end delays, we have proved a source coding theorem that shows that the distortion-rate bound is achievable. Furthermore, for estimation, it is possible to completely characterize the information being produced by an unstable process. [23] One part of the information captures the essential unstable nature and requires anytime reliability for transport across a noisy channel. As also discussed in this paper, anytime reliability is a sense of reliable transmission that lies between Shannon's classical $\epsilon$-sense of reliable transmission and his zero-error reliability.[28] The residual information in the open-loop process is purely classical in nature and determines the actual distortion performance achieved by the code. In [23], we also review how the sense of anytime reliability is linked to classical work on sequential tree codes with bounded delay decoding. ${ }^{2}$

The new feature in control systems is the essential interactivity of the problem. The information to be communicated is not a message known in advance that is used by some completely separate entity. Rather, it evolves through time and is used to control the very process being encoded. This introduces two interesting issues. First, causality is strictly enforced at the encoder. The encoder and controller must act in real time and so taking the limit of large delays must be interpreted very carefully. Second, it is unclear what the status of the controlled process is. If the controller succeeds in stabilizing the process, it is no longer unstable. As explored in section 3.5, a purely external non-interactive observer could treat the question of encoding the controlled closed-loop system state using classical tools for the encoding and communication of a stationary ergodic process. Despite having to observe and encode the exact same closed-loop process, the observer internal to the control system ends up requiring a channel as good as that required to communicate the unstable open-loop process. This seemingly paradoxical situation illustrates what can happen when the encoding of information and its use are coupled together by interactivity.

In part I, the basic equivalence between control and reliable communication is established. After a review of notation in section 2 , the scalar problem is formally introduced in section 3 where it is also established that classical concepts are inadequate to dealing with the problem. In section 4, we show that for a general sense of stability, that adequate feedback anytime capacity is necessary for there

\footnotetext{
${ }^{2}$ Reference [2] raised the possibility of such a connection early on.
} 
to exist any observer/controller pair able to stabilize the unstable system across the noisy channel. This connection is also exploited to give a sequential anytime version of the Schalkwijk/Kailath communication scheme for the AWGN channel with noiseless feedback. In section 5, we first show the sufficiency of adequate feedback anytime capacity for situations where the observer has noiseless access to the channel outputs. In section 6, these sufficiency results are then generalized to the case where the observer only has noisy access to the plant state. Since the necessary and sufficient conditions are tight in general, these results show the asymptotic equivalence between the problem of control with "noisy feedback" and the problem of reliable sequential communication with noiseless feedback. In section 7, these results are further extended to the continuous time scalar setting.

In part II (section 8), these results are generalized to the case of vector-valued state on an unstable eigenvalue by eigenvalue basis. The role of anytime capacity is played by a rate region corresponding to a vector of anytime reliabilities. For cases without explicit noiseless feedback of the channel outputs, the intrinsic delay of the control system's input-output behavior plays an important role. It shows that two systems with the same unstable eigenvalues can still have different channel requirements. In cases where the system matrices have non-diagonal Jordan blocks, successive decoding ${ }^{3}$ is used to prove the result.

These results establish that in interactive settings, a single "application" can fundamentally require different senses of reliability for its data streams. No single number can adequately summarize the channel and any layered communication architecture should allow applications to adjust reliabilities on bitstreams. Unlike our paper on the estimation of unstable processes[23], in this work we can make no statements about the performance achieved by the control system. All results here are only in terms of the binary question of stabilization, though we can make precise statements about various senses of stability.

\section{General Notation}

We use the shorthand $a_{1}^{t}=\left(a_{1}, a_{2}, \ldots, a_{t-1}, a_{t}\right)$ to refer to a sequence. We will also apply scalar functions to vectors on a component by component basis so $\log _{2} \vec{\lambda}$ is simply shorthand for the vector whose components are $\log _{2} \lambda_{i}$. Similarly $\vec{\lambda}_{\|}$is shorthand for the vector whose components are $\left|\lambda_{i}\right|$.

Notation used throughout the paper is given here and symbols used only in particular sections will be listed at the beginning of those sections.

$\mathcal{A}$ Channel input alphabet

$A$ The unstable system dynamics. Either scalar or square matrix depending on the context.

$a_{t}$ The channel input at time $t$

$\mathcal{B}$ Channel output alphabet

$B_{t}$ The random variable denoting the channel output at time $t$.

$p\left(B_{t} \mid a_{t}\right)$ The probability measure on memoryless channel output $B_{t}$ given that the input was $a_{t}$.

$C$ The Shannon capacity of the channel

\footnotetext{
${ }^{3}$ Inspired by how one can communicates in a degraded broadcast or multiple-access scenario where the strongest message is decoded first and then used to decode the next message.
} 
$C_{0}$ The Shannon zero-error capacity of the channel

$C_{\text {any }}(\alpha)$ The maximum rate at which the channel supports anytime reliability $\alpha$ from definition 4.2

$C_{\mathrm{g} \text {-any }}(g)$ The maximum rate at which the channel supports $g$-anytime reliability $g(d)$ from definition 4.4

$d$ The delay of interest. Measured in terms of channel uses whenever we are in discrete time.

$f(m)$ A decreasing function used to upper bound $P\left(\left|X_{t}\right|>m\right)$

$g(d)$ A decreasing function used to upper bound the probability of error on messages from more than $d$ time steps ago

$I(A ; B)$ Mutual information between random variables $A$ and $B$.

$i, j, k$ Integer valued indices used in various ways.

$K$ Some constant that we do not care about. Does not depend on delay $d$ or time $t$.

$\mathcal{P}$ The probability function. Unless specified, all probabilities are taken over all sources of randomness in the system.

$N_{t}$ The noise. In section 5.7, it refers to the bounded noise in the observation of $X_{t}$.

$n$ The block-length $n$ used when considering time in blocks.

$R$ The rate of communication in bits per channel use.

$m$ A dummy variable used when measuring the probability in the tail of a distribution.

$M_{t}$ The message to be communicated that has arrived at time $t$. It consists of all bits that have arrived so far and not already batched into $M_{t-1}$ or earlier messages.

$U_{t}$ The scalar control applied to the scalar system at the end of time $t$.

$W_{t}$ The bounded scalar disturbance entering the scalar system at the end of time $t$.

$X_{t}$ The scalar plant state at time $t$. The plant is assumed to evolve at the same time scale as the channel uses when in discrete time.

$\alpha$ The exponential anytime reliability from definition 4.1.

$\eta$ The real moment of $|X|$ that is desired to be kept finite by the control system. $\eta>0$

$\Omega$ The bound on how large the plant disturbance $W$ can be.

$\mathcal{D}$ The anytime decoder: produces updated estimates of all messages $\hat{M}_{1}^{t}(t)$ at time $t$.

$\mathcal{E}$ The anytime encoder: takes all messages and feedback information it has access to and produces a channel input at each time step.

$\mathcal{O}$ The plant observer: takes observations of the plant and any other information it has access to and produces a channel input at each time step.

$\mathcal{C}$ The plant controller: takes the channel outputs so far and produces a control signal to apply to the plant. 


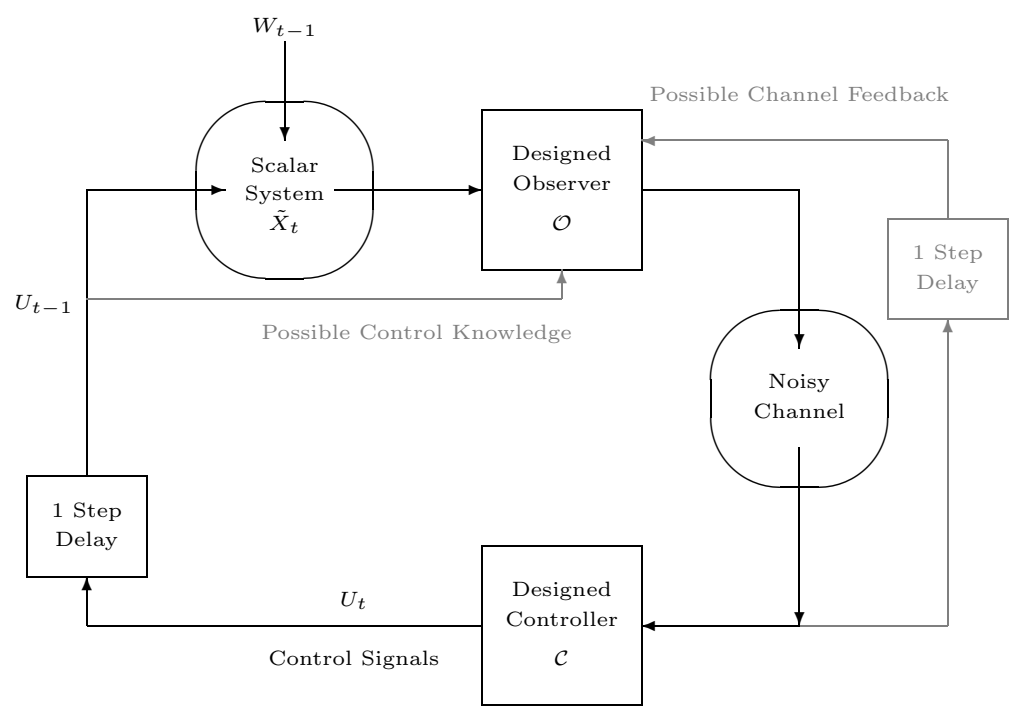

Figure 2: Control over a noisy communication channel. The unstable scalar system is persistently disturbed by $W_{t}$ and must be kept stable in closed-loop through the actions of $\mathcal{O}, \mathcal{C}$.

\section{Problem definition and basic challenges}

After reviewing notation, we formally introduce the control problem of stabilizing an unstable scalar linear system driven by both a control and a bounded disturbance in subsection 3.2. In subsection 3.3, classical notions of capacity are reviewed and the noiseless channel example used to show how it can be possible to stabilize an unstable system with finite rate. In 3.4, it is shown by example that the classical concepts are inadequate when it comes to evaluating a noisy channel for control purposes. Shannon's regular capacity is too optimistic and zero-error capacity is too pessimistic. Finally, section 3.5 shows that the core issue of interactivity is different than merely requiring the encoders and decoders to be delay-free.

\subsection{Notation specific to this section}

$\delta$ The probability of erasure in an erasure channel.

\subsection{The control problem}

$$
X_{t+1}=A X_{t}+U_{t}+W_{t}, \quad t \geq 0
$$

where $\left\{X_{t}\right\}$ is a $\mathbb{R}$-valued state process. $\left\{U_{t}\right\}$ is a $\mathbb{R}$-valued control process and $\left\{W_{t}\right\}$ is a bounded noise/disturbance process s.t. $\left|W_{t}\right| \leq \frac{\Omega}{2}$. This bound is assumed to hold almost surely. For convenience, we also assume a known initial condition ${ }^{4} X_{0}=0$.

\footnotetext{
${ }^{4}$ The perfectly known initial condition and bound on the noise/disturbance makes it easy to get the results, but some of it generalizes to Gaussian disturbances. For bounded initial conditions, just introduce a zero initial condition at time $X_{-1}$ and a special initial disturbance. The Gaussian case will be covered in subsequent work and some of the story here goes through because the tail of the Gaussian is very light. As such, it is almost bounded and occasional large values can be dealt with at the expense of a little variability in the rate of the code.
} 
To make things interesting, we consider $A>1$ so the open-loop system is exponentially unstable. The distributed nature of the problem comes from having a noisy communication channel in the feedback loop. We require an observer/encoder system $\mathcal{O}$ to observe $X_{t}$ and generate inputs $a_{t}$ to the channel. It may or may not have access to the control signals $U_{t}$ or past channel outputs $B_{t-1}$ as well. We also require a decoder $/$ controller $^{5}$ system $\mathcal{C}$ to observe channel outputs $B_{t}$ and generate control signals $U_{t}$. We allow both $\mathcal{O}, \mathcal{C}$ to have arbitrary memory and to be nonlinear in general.

Definition 3.1 A closed-loop dynamic system with state $X_{t}$ is $f$-stable if $\mathcal{P}\left(\left|X_{t}\right|>m\right)<f(m)$ for all $t \geq 0$.

This definition requires the probability of a large state value to be appropriately bounded. ${ }^{6}$ Since we model the channel as stochastic, it is often too much to require that the state is bounded almost surely. There might be rare sequences of channel noise that will cause the state to grow outside any boundary. ${ }^{7}$ A looser sense of stability is given by:

Definition 3.2 A closed-loop dynamic system with state $X_{t}$ is $\eta$-stable if there exists a constant $K$ s.t. $E\left[\left|X_{t}\right|^{\eta}\right] \leq K$ for all $t \geq 0$.

Rather than having to specify a specific target for the tail probability $f$, holding the $\eta$-moment within bounds is a way of keeping large deviations rare. The larger $\eta$ is, the more strongly we penalize very large deviations. The advantage of $\eta$-stability is that it will allow us to ignore constant factors and thus make sharp asymptotic statements. Furthermore, section 4.4 will show that for generic DMCs, no sense stronger than $\eta$-stability is feasible.

The goal in this paper is to find necessary and sufficient conditions on the noisy channel so that there can exist an observer $\mathcal{O}$ and controller $\mathcal{C}$ so that the closed loop system shown in figure 2 is stable in the sense of definition 3.1. The problem is considered under different information patterns corresponding to different assumptions about what is available at the observer $\mathcal{O}$. The controller is always assumed to just have access to the entire past history of channel outputs.

An intrinsic rate of information production can be associated with the system of (1). For discretetime linear systems, the intrinsic rate (in units of bits per time) equals the sum of the logarithm (base 2 ) of the unstable eigenvalues. [35] In the scalar case studied here, this is just $\log _{2} A$. This means that it is generically ${ }^{8}$ impossible to stabilize the system in any reasonable sense if the feedback channel's Shannon classical capacity $C<\log _{2} A$.

\footnotetext{
${ }^{5}$ Because the decoder and controller are both on the same side of the communication channel, we lump them together into a single box. Certain architectures for achieving control will of course have these in separate boxes.

${ }^{6}$ To avoid getting bogged down in notation, we are ignoring the issue of not necessarily having a probability distribution for the plant disturbance. References [19] and [18] deal with this explicitly by invoking a hypothetical observer which considers the distribution of an interval of uncertainty that contains the state. Alternatively, we can consider the requirement that the probability be bounded with a uniform bound over all possible disturbance sequences.

${ }^{7}$ Consider a network control model where packets might be erased with some probability. If the system is allowed to run for a long time, then it becomes increasingly certain that a run of bad luck on the channel will occur sometime.

${ }^{8}$ There are pathological cases where it is possible to stabilize a system with less rate. These occur when the driving disturbance is particularly structured instead of just being unknown but bounded. An example is when the disturbance only takes on values \pm 1 while $A=4$. Clearly only one bit per unit time is required even though $\log _{2} A=2$.
} 


\subsection{Classical notions of channels and capacity}

Definition 3.3 $A$ discrete time channel is a probabilistic system with an input. At every time step $t$, it takes an input $a_{t} \in \mathcal{A}$ and produces an output $b_{t} \in \mathcal{B}$ with probability ${ }^{9} p\left(B_{t} \mid a_{1}^{t}, b_{1}^{t-1}\right)$. In general, the current channel output is allowed to depend on all inputs so far as well as on past outputs.

The channel is memoryless if conditioned on $a_{t}, B_{t}$ is independent of any other random variable in the system that occurs at time $t$ or earlier. All that needs to be specified is $p\left(B_{t} \mid a_{t}\right)$.

The maximum rate achievable for a given sense of reliable communication is called the associated capacity. Shannon's classical reliability requires that after a suitably large end-to-end delay ${ }^{10} n$ that the average probability of error on a bit is below a specified $\epsilon$. Shannon classical capacity $C$ can also be calculated in the case of memoryless channels by solving an optimization problem:

$$
C=\sup _{\mathcal{P}(A)} I(A ; B)
$$

where the maximization is over the input probability distribution and $I(A ; B)$ represents the mutual information through the channel.[9] This is referred to as a single letter characterization of channel capacity for memoryless channels. There is another sense of reliability and its associated capacity called zero-error capacity which requires the probability of error to be exactly zero with sufficiently large $n$. It does not have a simple single-letter characterization.[28]

Example 3.1 Consider a system (1) with $\Omega=1$ and $A=\frac{3}{2}$. Target the second moment $\eta=2$ and suppose that the memoryless communication channel we face is a noiseless one bit channel. So $\mathcal{A}=\mathcal{B}=\{0,1\}$ and $p\left(B_{t}=1 \mid a_{t}=1\right)=p\left(B_{t}=0 \mid a_{t}=0\right)=1$ while $p\left(B_{t}=1 \mid a_{t}=0\right)=p\left(B_{t}=0 \mid a_{t}=\right.$ $1)=0$. This channel has $C_{0}=C=1>\log _{2} \frac{3}{2}$.

Use a memoryless observer

$$
\mathcal{O}(x)= \begin{cases}0 & \text { if } x \leq 0 \\ 1 & \text { if } x>0\end{cases}
$$

and memoryless controller

$$
\mathcal{C}(B)= \begin{cases}+\frac{3}{2} & \text { if } B=0 \\ -\frac{3}{2} & \text { if } x>0\end{cases}
$$

Assume that the closed loop system state is within the interval $[-2,+2]$. If it is positive, then it is in the interval $[0,+2]$. At the next time, $\frac{3}{2} X+W$ will be in the interval $\left[-\frac{1}{2}, \frac{7}{2}\right]$. The applied control of $-\frac{3}{2}$, will shift the state back to within the interval $[-2,+2]$. The same argument holds by symmetry on the negative side. Since it starts at 0 , by induction it will stay within $[-2,+2]$ forever. As a consequence, the second moment will also stay less than 4 for all time.

Notice that the same observers and controllers would not have worked over a binary symmetric channel with crossover probability $\epsilon$, no matter how small the crossover probability were. Eventually, we would end up applying the wrong control. At that point, the state could shift to the interval $[1,5]$. If the state ends up larger than 4, then no possible disturbance or further controls of $-\frac{3}{2}$ could ever bring it back to the neighborhood of the origin.

\footnotetext{
${ }^{9}$ This is a probability mass function in the case of discrete alphabets $\mathcal{B}$, but is more generally an appropriate probability measure over the output alphabet $\mathcal{B}$.

${ }^{10}$ Traditionally, the community has used block-length for a block code as the fundamental quantity rather than delay. It is easy to see that doing encoding and decoding in blocks of size $n$ corresponds to a delay of between $n$ and $2 n$ on the individual bits being communicated.
} 
In addition to the Shannon and zero-error senses of reliability, information theory has various reliability functions. Such reliability functions (or error exponents) are traditionally considered a matter purely internal to channel coding and they have served as mathematically tractable proxies for the issue of implementation complexity. [9] Reliability functions study how fast the probability of error goes to zero as we increase the relevant system parameter. As such, the reliability functions for block-codes are given in terms of the block length, reliability functions for convolutional codes in terms of the constraint length[10], and reliability functions for variable-length codes in terms of the expected block length.[3] With the rise of sparse code constructions and iterative decoding, the role of error exponents in channel coding has been diminished since such approximate decoders generally do not give exponentially small probabilities of error.

For memoryless channels, the presence or absence of feedback does not alter the classical Shannon capacity. [9] More surprisingly, for symmetric DMCs, the block coding reliability functions (error exponents) also do not change with feedback, at least in the high rate regime.[6] From a control perspective, this is our first indication that neither Shannon's capacity nor block-coding reliability functions are the perfect fit for control applications.

\subsection{Counterexample showing classical concepts are inadequate}

To construct our counterexample showing the inadequacy of the Shannon classical capacity in characterizing channels for control, we will consider erasure channels. While both erasure and AWGN channels are easy to deal with, it turns out that AWGN channels can not be used for a counterexample since they can be treated in the classical LQG framework.[1] The deeper reason for why AWGN channels do not result in a counterexample will be given in section 4.4 .

\subsubsection{Erasure channels}

The packet erasure channel models situations where errors can be reliably detected at the receiver. In the model, sometimes the packet being sent does not make it through with probability $\delta$, but otherwise it makes it through correctly. Explicitly:

Definition 3.4 The L-bit packet erasure channel is a memoryless channel with $\mathcal{A}=\{0,1\}^{L}, \mathcal{B}=$ $\{0,1\}^{L} \cup\{\emptyset\}$ and $p(x \mid x)=1-\delta$ while $p(\emptyset \mid x)=\delta$.

It is well known that the Shannon capacity of the packet erasure channel is $(1-\delta) L$ bits per channel use regardless of whether the encoder has feedback or not.[9] Furthermore, because a long string of erasures is always possible, the Shannon zero-error capacity of this channel is 0 as long as $\delta>0$. There are also variable-length packet erasure channels where the packet-length is something the encoder can choose. See [21] for a discussion of such channels.

To make our simple counterexample, we will consider a further abstraction:

Definition 3.5 The real packet erasure channel has $\mathcal{A}=\mathcal{B}=\mathbb{R}$ and $p(x \mid x)=1-\delta$ while $p(0 \mid x)=\delta$.

This represents a situation where the channel can carry a single real number per use, but sometimes the real number will be lost and replaced with a zero. This model has also been explored in the context of Kalman filtering with lossy observations.[31] It has infinite classical capacity since a single real number can carry arbitrarily many bits within its binary expansion. 


\subsubsection{The inadequacy of Shannon capacity}

The real erasure channel provides us with an easy way of seeing the inadequacy of Shannon capacity as a way of characterizing channels for control. We use the problem from example 3.1, except over the real erasure channel instead of the one bit noiseless channel. Recall that we want the second moment to be bounded $(\eta=2)$ and that $A=\frac{3}{2}$. Let $\delta=\frac{1}{2}$ so that there is a $50 \%$ chance of any real number being erased. For the driving noise/disturbance $W_{t}$, assume that it is zero-mean and i.i.d. with variance $\sigma^{2}$.

The classical capacity of the real erasure channel is $\infty>\log _{2} \frac{3}{2}$ since when the real number is received correctly, it can communicate an infinite number of bits correctly. Thus the basic necessary condition for stabilizability is met.

In this case, the optimal control is obvious - set $a_{t}=X_{t}$ as the channel input and use $U_{t}=-A B_{t}$ as the control. With every successful reception, the system state is reset to the initial condition of zero. For an arbitrary time $t$, the time since it was last reset is distributed like a $\frac{1}{2}$-geometric random variable ${ }^{11}$. Thus the second moment is:

$$
\begin{aligned}
E\left[\left|X_{t+1}\right|^{2}\right] & >\sum_{i=0}^{t} \frac{1}{2}\left(\frac{1}{2}\right)^{i} E\left[\left(\sum_{j=0}^{i}\left(\frac{3}{2}\right)^{j} W_{t-j}\right)^{2}\right] \\
& =\sum_{i=0}^{t} \frac{1}{2}\left(\frac{1}{2}\right)^{i} \sum_{j=0}^{i} \sum_{k=0}^{i}\left(\frac{3}{2}\right)^{j+k} E\left[W_{t-j} W_{t-k}\right] \\
& =\sum_{i=0}^{t}\left(\frac{1}{2}\right)^{i+1} \sum_{j=0}^{i}\left(\frac{9}{4}\right)^{j} \sigma^{2} \\
& =\frac{4 \sigma^{2}}{5} \sum_{i=0}^{t}\left(\left(\frac{9}{8}\right)^{i+1}-\left(\frac{1}{2}\right)^{i+1}\right)
\end{aligned}
$$

This diverges as $t \rightarrow \infty$ since $\frac{9}{8}>1$.

Notice that the root of the problem is that $\left(\frac{3}{2}\right)^{2}\left(\frac{1}{2}\right)>1$. Intuitively, the system is getting away from us faster than the noisy channel is able to give us reliability. This causes the second moment to explode. If we were instead concerned with the first moment $E\left[\left|X_{t}\right|\right]$, then it would be bounded for all $t$ since $\left(\frac{3}{2}\right)\left(\frac{1}{2}\right)<1$.

The adequacy of the channel depends on which moment we want to be bounded. Thus no singlenumber characterization like classical capacity can give us the figure-of-merit that we need to evaluate the channel for control applications.

\subsection{Non-interactive observation of closed-loop process}

Consider the problem shown in figure 3. In this, we have added an additional passive joint sourcechannel encoder $\mathcal{E}^{s}$ watching the closed loop state $X_{t}$ and communicating it to a passive estimator $\mathcal{D}^{s}$ through a second independent noisy channel. Both the passive and internal observers have access to the same plant state and to be fair, we will also require the passive encoder and decoder to be causal - no end-to-end delay is permitted. At first glance, it certainly appears that the communication

\footnotetext{
${ }^{11}$ This is the process that models the time till first head in a sequence of fair coin tosses.
} 


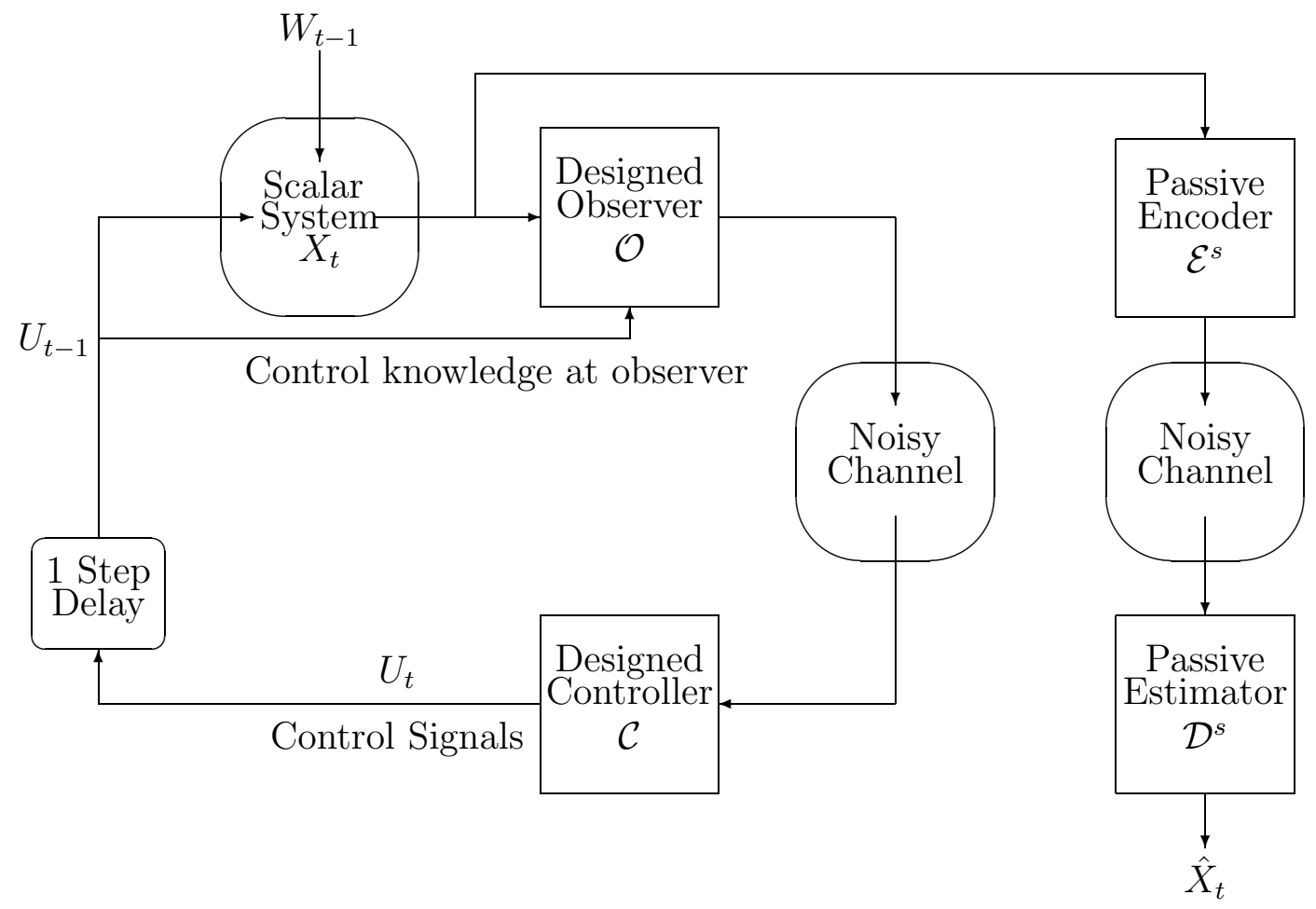

Figure 3: The control system with an additional passive joint source-channel encoder $\mathcal{E}^{s}$ watching the closed loop state $X_{t}$ and communicating it to a passive estimator $\mathcal{D}^{s}$. The controller $\mathcal{C}$ implicitly needs a good causal estimate for $X_{t}$ and the passive estimator $\mathcal{D}^{s}$ explicitly needs the same thing. Which requires the better channel? 
situations are symmetric. If anything, the internal observer is better off since it also has access to the control signals while the passive observer is denied access to them.

Suppose that the closed-loop process (1) had already been stabilized by the observer and controller system of 3.1, so that the second moment $E\left[X_{t}^{2}\right] \leq K$ for all $t$. Suppose that the noisy channel facing the passive encoder is the real erasure channel of the previous section. We can apply the same observation rule, and ask how well the passive observer does at estimating this process.

The optimal encoding rule is clear, set $a_{t}=X_{t}$. It is certainly feasible, though not optimal ${ }^{12}$, to use $\hat{X}_{t}=B_{t}$ itself as our estimator for the process. This passive observation system clearly achieves $E\left[\left(\hat{X}_{t}-X_{t}\right)^{2}\right] \leq \frac{K}{2}<K$ since the probability of non-erasure is $\frac{1}{2}$. The causal decoding rule is able to achieve a finite end-to-end squared error distortion over this noisy channel in a causal and memoryless way.

This example makes it clear that the challenge here is arising from interactivity, not simply being forced to be delay-free. The passive external encoder and decoder do not have to face the unstable nature of the source while the internal observer and controller do. An error made while estimating $\hat{X}_{t}$ by the passive decoder has no consequence for the next state $X_{t+1}$ while a similar error by the controller does.

\section{Anytime capacity and its necessity}

Anytime reliability is introduced and related to classical notions of reliability in [23]. Here, we will review the definitions, but will motivate them from the control point of view. As such, we will focus on the maximum rate achievable for a given sense of reliability rather than the maximum reliability possible at a given rate. The two are of course related since fundamentally there is an underlying region of feasible rate/reliability pairs.

Since the uncontrolled system state has the potential to grow exponentially, the controller's knowledge of the past must become certain at a fast rate in order to prevent a bad decision made in the past from continuing to corrupt the future. When viewed in the context of reliably communicating bits from an encoder to a decoder, this suggests that the estimates of the bits at the decoder must become increasingly reliable with time. After reviewing notation, the sense of anytime reliability is made precise in section 4.2. Section 4.3 then establishes the key result of this paper relating the problem of stabilization to the reliable communication of messages in the anytime sense. Finally, we study some consequences of this connection in section 4.4. Among these consequences is a sequential generalization of the Schalkwijk/Kailath scheme for communication over an AWGN channel that achieves a doubly-exponential convergence to zero of the probability of bit error for all delays simultaneously.

\subsection{Notation specific to this section}

$I_{t}$ A dummy variable used to denote the real-valued input to the bit extraction algorithm used to estimate bits from a real number.

$P, P^{\prime}$ The average and normalized average channel input power constraints for the AWGN example.

\footnotetext{
${ }^{12}$ The best thing to do is to use the conditional mean $\hat{X}_{t}=E\left[X_{t} \mid b_{1}^{t}\right]$ since we are looking to minimize the mean squared error.
} 
$P^{\prime \prime}$ The minimum average normalized power corresponding to the $A$ being used for the AWGN example. $P^{\prime \prime}<P^{\prime}$

$S_{i}$ The $i$-th bit to be communicated. It is considered to have been generated at time $\frac{i}{R}$.

$\bar{S}$ A hypothetical bitstream that differs from the true bitstream $S$ being communicated.

$\hat{S}_{i}(t)$ The decoder's best estimate for the value of bit $i$ after receiving channel outputs up through time $i$.

$T_{i}$ The threshold used to extract the estimate for bit $i$ from a real number.

$\tilde{X}_{t}$ An open loop version of the system that is driven only by the control signals $U$, but not the disturbance $W$. Defined in (3).

$\check{X}_{t}$ An open loop version of the system that is driven only by the disturbance $W$, but not the controls $U$. Defined in (4).

$X_{t}^{w}$ The closed loop plant state $X$ for the AWGN example driven only by the disturbance $W$ with the channel noise set to zero.

$\beta$ The scalar gain used in the linear observer for the AWGN example.

$\phi$ The scalar gain used in the linear controller for the AWGN example.

$\epsilon_{1}$ The slack used in encoding bits into a real number. $\left(2+\epsilon_{1}\right)$ is the "base" of the Cantor-set encoding. The choice for $\epsilon_{1}$ is given in (7).

$\gamma$ A constant chosen to meet the disturbance bound in the encoding of bits into a real number. The choice for $\gamma$ is given in (8).

$\omega$ A member of the sample space of the underlying probability space.

$\sigma^{2}$ The variance of the AWGN channel noise.

$\sigma_{x}^{2}$ The variance of the plant state for the AWGN example when the disturbance $W$ is not present.

\subsection{Anytime reliability and capacity}

We will operate in the context of sequential codes. The entire message is not assumed to be known ahead of time. Rather, it is made available to us as time evolves. For simplicity of notation, let $M_{i}$ be the $R$ bit message that the channel encoder gets at time $i$. We assume that the channel decoder does not necessarily know when the message $i$ will be needed by the application. A past message may also be needed more than once by the application. As such, the anytime decoder produces estimates $\hat{M}_{i}(t)$ which are the best estimates for message $i$ at time $t$ based on all the channel outputs received so far. If we are using the past messages with a delay $d$, the probability of error we are interested in is $\mathcal{P}\left(\hat{M}_{1}^{t-d} \neq M_{1}^{t-d}\right)$. This corresponds to an uncorrected error anywhere in the distant past beyond $d$ channel uses ago.

Definition 4.1 A rate $R$ communication system over a noisy channel is an encoder $\mathcal{E}$ and decoder $\mathcal{D}$ pair such that: 
- $R$-bit message $M_{i}$ enters $^{13}$ the encoder at time $i$

- The encoder produces a channel input at integer times based on all information that it has seen so far. For encoders with access to feedback with delay $1+\theta$, this also includes the past channel outputs $B_{1}^{t-1-\theta}$.

- The decoder produces updated channel estimates $\hat{M}_{i}(t)$ for all $i \leq t$ based on all channel outputs observed till time $t$

A rate $R$ sequential communication system achieves anytime reliability $\alpha$ if there exists a constant $K$ such that:

$$
\mathcal{P}\left(\hat{M}_{1}^{i}(t) \neq M_{1}^{i}\right) \leq K 2^{-\alpha(t-i)}
$$

holds for every $i, t$. The probability is taken over the channel noise, the $R$ bit messages ${ }^{14} M_{i}$, and all of the common randomness available in the system.

If (2) holds for every possible realization of the messages $M$, then we say that the system achieves uniform anytime reliability $\alpha$.

Communication systems that achieve anytime reliability are called anytime codes and similarly for uniform anytime codes.

We could alternatively have bounded the probability of error by $2^{-\alpha\left(d-\log _{2} K\right)}$ and interpreted $\log _{2} K$ as the minimum delay imposed by the communication system. The asymptotic aspect of the definition comes from our lack of concern about the minimum delay $\log _{2} K$ required.

Definition 4.2 The $\alpha$-anytime capacity $C_{a n y}(\alpha)$ of a channel is the least upper bound of the rates $R$ (in bits) at which the channel can be used to construct a rate $R$ communication system that achieves uniform anytime reliability $\alpha$.

Feedback anytime capacity is used to refer to the anytime capacity when the encoder has access to noiseless feedback of the channel outputs with unit delay.

The requirement for exponential decay in the probability of error with delay is reminiscent of the block-coding reliability functions $E(R)$ of a channel given in [9]. There is one crucial difference. In the standard study of error exponents, both the encoder and decoder vary with blocklength or delay $n$. In our definition of $\alpha$-anytime capacity, we require the encoding to be fixed and the decoder has to in principle work at all delays since we are considering estimates of the message $M_{i}$ at all times $t>i$.

This additional requirement is why we call it "anytime" capacity. We can query the decoding process for a given bit at any time and we require the answer to be increasingly meaningful the longer we wait. The anytime $\alpha$ specifies the exponential rate at which we want the answers to improve. The above sense of reliable transmission lies between that represented by classical zeroerror capacity (probability of error becomes zero at a large but finite delay) and classical capacity (probability of error becomes something small at a large but finite delay). It should be clear that $\forall \alpha, C_{0} \leq C_{\text {any }}(\alpha) \leq C$.

\footnotetext{
${ }^{13}$ In what follows, we will often consider single bit messages for simplicity of exposition. In that case, the $i$-th bit arrives at the encoder at time $\frac{i}{R}$.

${ }^{14}$ In the definition, we gloss over the issue of non-integer values of $R$. These can be interpreted in many ways. The easiest is to consider that the size of the alphabet for the messages $M$ might change in a regular pattern from time to time. This is not a serious issue.
} 
By using a random coding argument over infinite tree codes, it is possible to show the existence of anytime codes without using feedback between the encoder and decoder for all rates less than the Shannon capacity. This shows:

$$
C_{\text {any }}\left(E_{r}(R)\right) \geq R
$$

where $E_{r}(R)$ is Gallager's random coding error exponent calculated in base 2 and $R$ is the rate in bits.[19, 23] Since feedback plays an essential role in control, it turns out that we are interested in the anytime capacity when feedback is available to the encoder. Just as the error exponent can sometimes be larger with feedback, the anytime reliability can as well.[19]

\subsection{Necessity of anytime capacity}

Anytime reliability and capacity are defined in terms of bits or digital messages that must be reliably communicated from point to point. Stability is a notion involving the analog value of the state of a plant in interaction with a controller over a noisy feedback channel. At first glance, these two problems appear to have nothing in common with each other except perhaps the noisy channel. Even on that point there is a difference. The observer/encoder $\mathcal{O}$ in the control system may have no explicit access to the noisy output of the channel. It can appear to be using the noisy channel without feedback. In this section, we will show that the relevant digital communication problem will involve access to the noisy channel with noiseless feedback coming back to the message encoder.

The key result is:

Theorem 4.3 For a given noisy channel and $\eta>0$, if there exists an observer $\mathcal{O}$ (with or without channel feedback or access to the controls) and controller $\mathcal{C}$ for the unstable scalar system that achieves $E\left[\left|X_{t}\right|^{\eta}\right]<K$ for all sequences of bounded driving noise $\left|W_{t}\right| \leq \frac{\Omega}{2}$, then $C_{\text {any }}\left(\eta \log _{2} A\right) \geq \log _{2} A$ bits per channel use for the noisy channel considered with the encoder having access to noiseless feedback.

Assume that we have an observer/controller pair $(\mathcal{O}, \mathcal{C})$ that can stabilize the unstable system. In the following sections, we show how to use that pair to construct a rate $R<\log _{2} A$ anytime encoder and decoder for a channel with noiseless feedback by using the observer/controller and wrapping them around a simulated plant. This will constructively show the necessity of anytime capacity. ${ }^{15}$

The strategy is illustrated in figure 4 . The key is to think of the simulated plant state as the sum of the states of two different unstable LTI systems. The first, with state denoted $\tilde{X}_{t}$, is driven entirely by the controls and starts in state 0 .

$$
\tilde{X}_{t+1}=A \tilde{X}_{t}+U_{t}
$$

These controls are available at the encoder due to the presence of noiseless feedback. ${ }^{16}$ The other, with state denoted $\check{X}_{t}$, is driven entirely by a simulated driving noise that is generated from the data stream we want to communicate.

$$
\check{X}_{t+1}=A \check{X}_{t}+W_{t}
$$

The sum $X_{t}=\left(\tilde{X}_{t}+\check{X}_{t}\right)$ behaves exactly like it was coming from (1) and is fed to the observer which uses it to generate inputs for the noisy channel.

\footnotetext{
${ }^{15}$ In traditional rate-distortion theory, this direction is shown by going through the mutual information characterizations of both the rate-distortion function and the channel capacity function. In our case, mutual information is not discriminating enough and so we have to prove the theorem directly.

${ }^{16}$ The encoder can run a copy of the controller and feed it the noiseless feedback to get the controls. If the controller is randomized, then we require this to be common randomness shared by the encoder and decoder.
} 


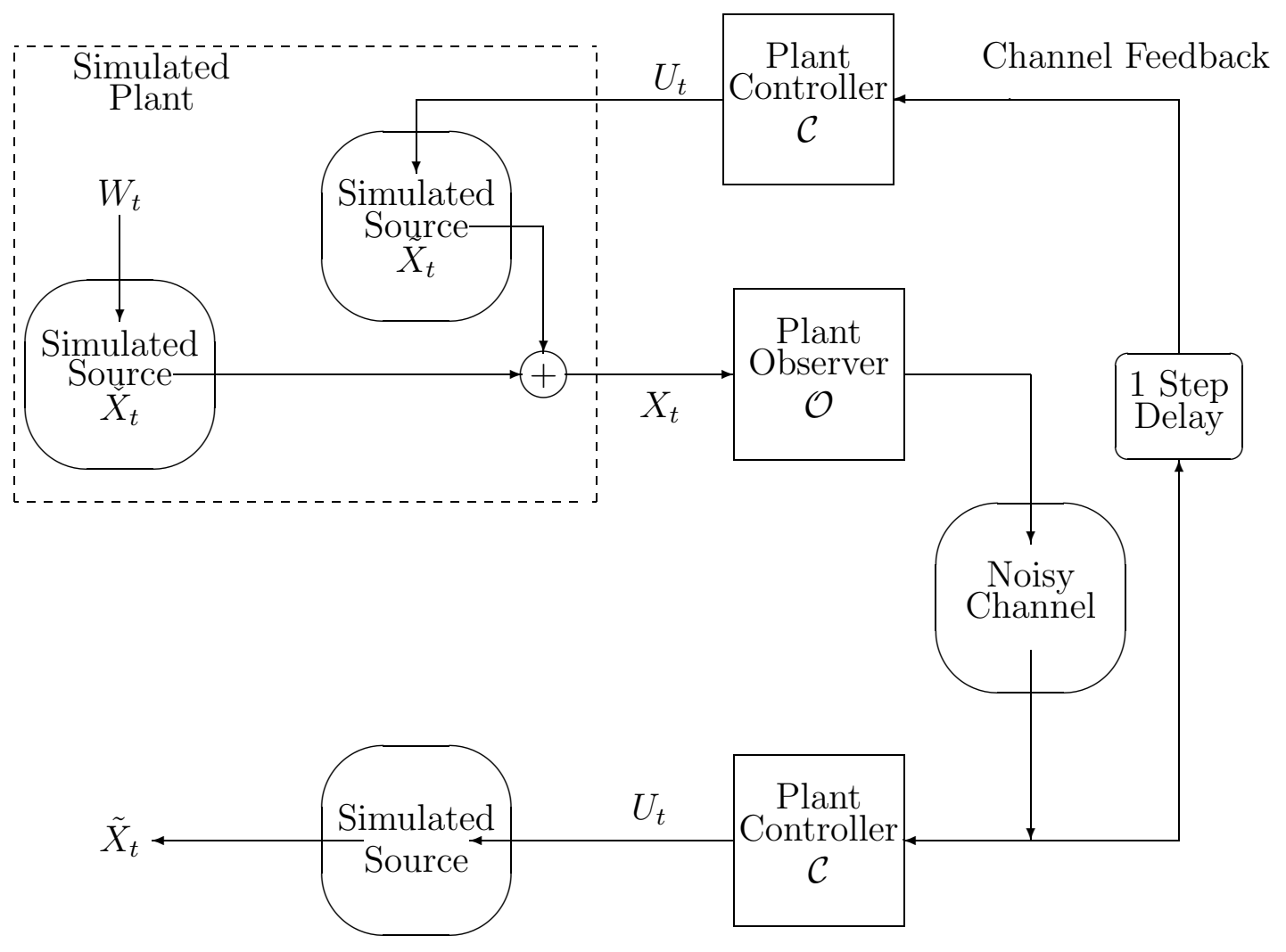

Figure 4: How to construct a simulated unstable open-loop source with access to noiseless feedback so that the decoder gets access to a good estimate of the unstable source. The data to be communicated is used to generate the appropriate $W$ inputs at the encoder. These data bits are then extracted from the $\tilde{X}$ at the decoder. 
The decoder runs its own copy of the $\tilde{X}_{t}$ process based on the controls $U_{t}$ which are available to it from the controller using the outputs from the noisy channel. The fact that the original observer/controller pair stabilized the original system implies that $\left|X_{t}\right|=\left|\check{X}-\left(-\tilde{X}_{t}\right)\right|$ is small and hence $-\tilde{X}_{t}$ stays close to $\check{X}_{t}$. All we need to do now is to show how to encode our message into the $W_{t}$, extract our past messages from $\left(-\tilde{X}_{t}\right)$, and bound the probability of error.

\subsubsection{Encoding data into the state}

The model of the disturbance is unknown but bounded. As such, we are allowed to choose any disturbance we like ${ }^{17}$ for the simulated plant. The choice will be determined by the data rate $R$ and the actual message to be sent. Rather than working with messages $M_{i}$, we will focus on a bitstream $S_{i}$ where bit $i$ becomes available to us at time $\frac{i}{R}$. Everything here can generalize naturally to non-binary alphabets for the message, but it is convenient to focus on the binary case.

$\check{X}_{t}$ is the part of $X_{t}$ that only has the driving noise as its input.

$$
\begin{aligned}
\check{X}_{t} & =A \check{X}_{t-1}+W_{t-1} \\
& =\sum_{i=0}^{t-1} A^{i} W_{t-1-i} \\
& =A^{t-1} \sum_{j=0}^{t-1} A^{-j} W_{j}
\end{aligned}
$$

This looks like the representation of a fractional number in base $A$ which is then multiplied by $A^{t-1}$. We will exploit this in our encoding by choosing the bounded disturbance sequence so that we have $^{18}$

$$
\check{X}_{t}=\gamma A^{t} \sum_{k=0}^{\lfloor R t\rfloor}\left(2+\epsilon_{1}\right)^{-k} S_{k}
$$

where $S_{k}$ is the $k$-th bit $^{19}$ of data that the anytime encoder has to send and $\lfloor R t\rfloor$ is just the total count of bits that are ready to be sent by time $t . \gamma$ is a constant chosen so as to meet the required bound on the simulated disturbance $W$.

To see that (5) is always possible to achieve by appropriate choice of bounded disturbance, use induction. (5) clearly holds for $t=0$. Now assume that it holds for time $t$ and consider time $t+1$ :

$$
\begin{aligned}
\check{X}_{t+1} & =A \check{X}_{t}+W_{t} \\
& =\gamma A^{t+1}\left(\sum_{k=0}^{\lfloor R t\rfloor}\left(2+\epsilon_{1}\right)^{-k} S_{k}\right)+W_{t}
\end{aligned}
$$

\footnotetext{
${ }^{17}$ In [23], a similar strategy was followed for when we assumed that we had a density for the i.i.d. disturbance $W_{t}$. In that context, it was important to choose a simulated disturbance sequence that behaved stochastically like $W_{t}$. This was accomplished by using common randomness shared between the encoder and decoder to shape the kind of disturbances produced here into ones that looked like $W_{t}$.

${ }^{18}$ For a rough understanding of the result, ignore the $\epsilon_{1}$ and suppose that we just encoded our message in binary. It is intuitive that any good estimate of the $\check{X}_{t}$ state is going to agree with $\check{X}_{t}$ in all the high order bits. Since the system is unstable, all the encoded bits will eventually become high-order bits as time goes on. So no bit error can persist for too long and still keep the estimate close to $\check{X}_{t}$. The $\epsilon_{1}$ in the encoding is a technical device to make this reasoning hold uniformly for all bit strings, rather than merely "typical" ones. This is important since we are aiming for exponentially small bounds and so can not neglect rare events.

${ }^{19}$ For the next section, it is convenient to have the disturbances balanced around zero and so we choose to represent the bit $S_{i}$ as +1 or -1 rather than the usual 1 or 0 .
} 
So setting

$$
W_{t}=\gamma A^{t+1} \sum_{k=\lfloor R t\rfloor+1}^{\lfloor R(t+1)\rfloor}\left(2+\epsilon_{1}\right)^{-k} S_{k}
$$

gives the desired result. To see that this can be made to meet the bound, we manipulate (6):

$$
\begin{aligned}
W_{t} & =\gamma A^{t+1}\left(2+\epsilon_{1}\right)^{-\lfloor R t\rfloor} \sum_{j=1}^{\lfloor R(t+1)\rfloor-\lfloor R t\rfloor}\left(2+\epsilon_{1}\right)^{-j} S_{\lfloor R t\rfloor+j} \\
& =\gamma A \frac{\left(2+\epsilon_{1}\right)^{R t-(\lfloor R t\rfloor)}}{A^{-t\left(1-R \frac{\log _{2}\left(2+\epsilon_{1}\right)}{\log _{2} A}\right)}} \sum_{j=1}^{\lfloor R(t+1)\rfloor-\lfloor R t\rfloor}\left(2+\epsilon_{1}\right)^{-j} S_{\lfloor R t\rfloor+j}
\end{aligned}
$$

To keep this bounded, we choose

$$
\epsilon_{1}=2^{\frac{\log _{2} A}{R}}-2
$$

which is strictly positive if $R<\log _{2} A$. Applying that substitution we have

$$
\begin{aligned}
\left|W_{t}\right| & =\left|\gamma A\left(2+\epsilon_{1}\right)^{R t-(\lfloor R t\rfloor)} \sum_{j=1}^{\lfloor R(t+1)\rfloor-\lfloor R t\rfloor}\left(2+\epsilon_{1}\right)^{-j} S_{\lfloor R t\rfloor+j}\right| \\
& <\left|\gamma A\left(2+\epsilon_{1}\right)\right| \\
& =\left|\gamma A^{1+\frac{1}{R}}\right|
\end{aligned}
$$

So by choosing

$$
\gamma=\frac{\Omega}{2 A^{1+\frac{1}{R}}}
$$

we are guaranteed to stay within the specified bound on the driving noise/disturbance.

\subsubsection{Extracting data bits from the state estimate}

Lemma 4.1 Given a channel with access to noiseless feedback, for all rates $R<\log _{2} A$, it is possible to encode bits into the simulated scalar plant so that the uncontrolled process behaves like (5) by using disturbances given in (6) and the formulas in (7) and (8). At the output end of the noisy channel, we can extract estimates $\hat{S}_{i}(t)$ for the $i$-th bit sent for which the error event

$$
\left\{\omega \mid \exists i \leq j, \hat{S}_{i}(t) \neq S_{i}(t)\right\} \subseteq\left\{\omega|| X_{t} \mid \geq A^{t-\frac{j}{R}}\left(\frac{\gamma \epsilon_{1}}{1+\epsilon_{1}}\right)\right\}
$$

and thus:

$$
\mathcal{P}\left(\hat{S}_{1}^{j}(t) \neq S_{1}^{j}(t)\right) \leq \mathcal{P}\left(\left|X_{t}\right| \geq A^{t-\frac{j}{R}}\left(\frac{\gamma \epsilon_{1}}{1+\epsilon_{1}}\right)\right)
$$

Proof: Here we use $\omega$ to denote members of the underlying sample space. ${ }^{20}$

At the decoder, we have $-\tilde{X}_{t}=\check{X}_{t}-X_{t}$ which is close to $\check{X}$ since $X_{t}$ is small. To see how to extract bits from $-\tilde{X}_{t}$, we first consider how to recursively extract those bits from $\check{X}_{t}$.

\footnotetext{
${ }^{20}$ If the bits to be sent are deterministic, this is the sample space giving channel noise realizations.
} 


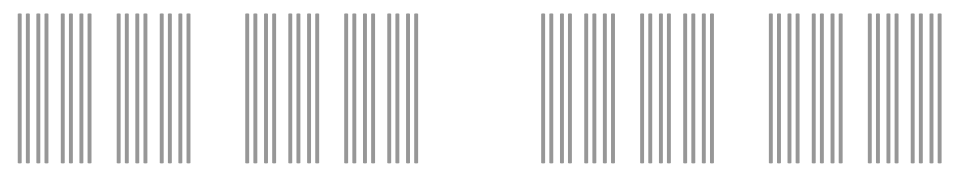

Figure 5: The data bits are used to sequentially refine a point on a Cantor set. The natural tree structure of the Cantor set construction allows us to encode bits sequentially. The Cantor set also has finite gaps between all points corresponding to bit sequences that first differ in a particular bit position. These gaps allow us to reliably extract bit values from noisy observations of the Cantor set point regardless of which point it is.

Starting with the first bit, we notice that the set of all possible $\check{X}_{t}$ that have $S_{0}=+1$ is separated from the set of all possible $\check{X}_{t}$ that have $S_{0}=-1$ by a gap of

$$
\begin{aligned}
& \gamma A^{t}\left(\left(1-\sum_{k=1}^{\lfloor R t\rfloor}\left(2+\epsilon_{1}\right)^{-k}\right)-\left(-1+\sum_{k=1}^{\lfloor R t\rfloor}\left(2+\epsilon_{1}\right)^{-k}\right)\right) \\
> & \gamma A^{t} 2\left(1-\sum_{k=1}^{\infty}\left(2+\epsilon_{1}\right)^{-k}\right) \\
= & \gamma A^{t} 2\left(1-\frac{1}{1+\epsilon_{1}}\right) \\
= & A^{t}\left(\frac{2 \epsilon_{1} \gamma}{1+\epsilon_{1}}\right)
\end{aligned}
$$

Notice that this worst-case gap ${ }^{21}$ is a positive number that is growing exponentially in $t$. If the first $i-1$ bits are the same, then we can scale both sides by $\left(2+\epsilon_{1}\right)^{i}=A^{\frac{i}{R}}$ to get the same expressions above and so by induction, it quickly follows that the minimum gap between the encoded state corresponding to two sequences of bits that first differ in bit position $i$ is given by

$$
\operatorname{gap}_{i}(t)=\inf _{\bar{S}: \bar{S}_{i} \neq S_{i}}\left|\check{X}_{t}(S)-\check{X}_{t}(\bar{S})\right|> \begin{cases}A^{t-\frac{i}{R}}\left(\frac{2 \gamma \epsilon_{1}}{1+\epsilon_{1}}\right) & \text { if } i \leq\lfloor R t\rfloor \\ 0 & \text { otherwise }\end{cases}
$$

Because the gaps are all positive, (11) shows that it is always possible to perfectly extract our data bits from $\check{X}_{t}$ by using an iterative procedure. ${ }^{22}$ To extract bit information from an input $I_{t}$ :

1. Initialize threshold $T_{0}=0$ and counter $i=0$.

2. Compare input $I_{t}$ to $T_{i}$. If $I_{t} \geq T_{i}$, set $\hat{S}_{i}(t)=+1$. If $I_{t}<T_{i}$, set $\hat{S}_{i}(t)=-1$.

3. Increment counter $i$ and update threshold $T_{i}=\gamma A^{t} \sum_{k=0}^{i-1}\left(2+\epsilon_{1}\right)^{-k} \hat{S}_{k}$

4. Goto step 2 as long as $i \leq\lfloor R t\rfloor$

Since the gaps given by (11) are always positive, the procedure works perfectly if we use input $I_{t}=\check{X}_{t}$. At the decoder, we will use $I_{t}=-\tilde{X}_{t}$.

\footnotetext{
${ }^{21}$ The typical gap is much larger and so the probability of error is actually much lower than this bound says it is.

${ }^{22}$ This is a minor twist on the procedure followed by serial A/D converters.
} 
With this, (9) is easy to verify by looking at the complementary event $\left\{\omega|| X_{t} \mid<\frac{A^{t-\frac{j}{R}} \gamma \epsilon_{1}}{1+\epsilon_{1}}\right\}$ By the bound (11) we are less than halfway across the minimum gap for bit $j$ at time $t$. As such, we can not make an error in the comparison in step 2 of the bit extraction procedure at iterations $i \leq j$.

\subsubsection{Probability of error for bounded moment and other senses of stability}

We are now ready to prove the main theorems.

Proof of Theorem 4.3: Using Markov's inequality we have:

$$
\begin{aligned}
\mathcal{P}\left(\left|X_{t}\right|>m\right) & =\mathcal{P}\left(\left|X_{t}\right|^{\eta}>m^{\eta}\right) \\
& \leq E\left[\left|X_{t}\right|^{\eta}\right] m^{-\eta} \\
& <K m^{-\eta}
\end{aligned}
$$

Combining with lemma 4.1, we get:

$$
\begin{aligned}
\mathcal{P}\left(\hat{S}_{1}^{i}(t) \neq S_{1}^{i}(t)\right) & \leq \mathcal{P}\left(\left|X_{t}\right| \geq A^{t-\frac{i}{R}}\left(\frac{\gamma \epsilon_{1}}{1+\epsilon_{1}}\right)\right) \\
& <K\left(\frac{1}{\gamma}+\frac{1}{\gamma \epsilon_{1}}\right)^{\eta} A^{-\eta\left(t-\frac{i}{R}\right)} \\
& =\left(K\left(\frac{1}{\gamma}+\frac{1}{\gamma \epsilon_{1}}\right)^{\eta}\right) 2^{-\left(\eta \log _{2} A\right)\left(t-\frac{i}{R}\right)}
\end{aligned}
$$

Since $t-\frac{i}{R}$ represents the delay between the time that bit $i$ was ready to be sent and the time at which we are doing the decoding, we are done.

All we needed from the bounded moment sense of stability was that it gave us some bound on the probability that $X_{t}$ took on large values. We can immediately generalize the proof above to other senses of stochastic stability and suitably generalize the sense of anytime capacity to allow for other bounds on the probability of error with delay.

Definition 4.4 A rate $R$ communication system achieves $g$-anytime reliability given by a function $g(d)$ if

$$
\mathcal{P}\left(\hat{M}_{1}^{t-d}(t) \neq M_{1}^{t-d}(t)\right)<g(d)
$$

The $g$-anytime capacity $C_{g \text {-any }}(g)$ of a noisy channel is the least upper bound of the rates $R$ at which the channel can be used to construct a sequential communication system that achieves $g$-anytime reliability given by the function $g(d)$.

Notice that for $\alpha$-anytime capacity, $g(d)=K 2^{-\alpha d}$ for some $K$.

Theorem 4.5 For a given noisy channel and decreasing function $f(m)$, if there exists an observer $\mathcal{O}$ and controller $\mathcal{C}$ for the unstable scalar system that achieves $\mathcal{P}\left(\left|X_{t}\right|>m\right)<f(m)$ for all sequences of bounded driving noise $\left|W_{t}\right| \leq \frac{\Omega}{2}$, then $C_{g \text {-any }}(g) \geq \log _{2} A$ for the noisy channel considered with the encoder having access to noiseless feedback and $g(d)$ having the form $g(d)=f\left(K A^{d}\right)$ for some constant $K$. 
Proof: For all rates $R<\log _{2} A$,

$$
\begin{aligned}
\mathcal{P}\left(\hat{S}_{1}^{i}(t) \neq S_{1}^{i}(t)\right) & \leq \mathcal{P}\left(\left|X_{t}\right| \geq \frac{A^{t-\frac{i}{R}} \gamma \epsilon_{1}}{1+\epsilon_{1}}\right) \\
& =f\left(\frac{\gamma \epsilon_{1}}{1+\epsilon_{1}} A^{t-\frac{i}{R}}\right)
\end{aligned}
$$

Since the delay $d=t-\frac{i}{R}$ we are done.

\subsection{Implications}

At this point, it is interesting to consider a few implications of theorem 4.5. First, we consider the possibility of more lax senses of stabilization and what is necessary to achieve them. Next, we show that for general DMCs it is impossible to have much stronger senses of stabilization without requiring zero-error capacity. The AWGN channel with an average input power constraint is considered next and we show how theorem 4.5 leads directly to a natural "anytime" generalization of the Schalkwijk/Kailath scheme.

\subsubsection{Weaker senses of stabilization than $\eta$-moment}

If all that we desire is that the probability of large deviation $\mathcal{P}\left(\left|X_{t}\right|>m\right) \leq f(m)$ where $f(m)$ goes to zero with large $m$, then we can take the limit of $C_{\text {any }}(\alpha)$ as $\alpha \downarrow 0$. We have shown elsewhere[19,23] that:

$$
\lim _{\alpha \downarrow 0} C_{\text {any }}(\alpha)=C
$$

where $C$ is the Shannon classical capacity. This holds for all discrete memoryless channels since the $\alpha$-anytime reliability goes to zero at Shannon capacity but is $>0$ for all lower rates even without feedback being available at the encoder.

It is interesting to also consider the possibility of having an even more lax sense of stability. For example, we could consider cases where $\lim _{m \rightarrow \infty} f(m)=10^{-5}>0$ which merely imposes the constraint that the probability of a large state stays below $10^{-5}$ for all time. Theorem 4.5 merely tells us that the probability of error is less than $10^{-5}$. However, Wolfowitz' converse to the coding theorem[9] tells us that since the block-length in this case is effectively going to infinity, we still require the Shannon capacity of the noisy channel to satisfy $C \geq \log _{2} A$. Adding a finite tolerance for arbitrarily large state values does not get around the need for the ability to communicate $\log _{2} A$ bits reliably.

\subsubsection{Stronger senses of stability than $\eta$-moment}

Having $f$ decrease only as a power law might not be suitable for certain applications. However, in general, this is all that we can hope for. Consider a DMC with no zero entries in its transition matrix. Define $\rho=\min _{i, j} p(i, j)$. For such a channel, with or without feedback, the probability of error after $d$ time steps is lower bounded by $\rho^{d}$ since that lower bounds the probability of all channel output sequences of length $d .{ }^{23}$ This implies that the probability of error can drop no more than exponentially in $d$ for such DMCs.

\footnotetext{
${ }^{23}$ Better upper-bounds on anytime reliability with feedback are possible. Reference [30] has such a bound that mirrors the convolutional coding exponent with constraint length.
} 
Theorem 4.5 therefore implies that the only $f$-senses of stability which are possible over such channels are those for which:

$$
\begin{aligned}
f\left(K A^{d}\right) & \geq \rho^{d} \\
f(m) & \geq \rho^{\frac{\log _{2}\left(\frac{m}{K}\right)}{\log _{2} A}} \\
f(m) & \geq K^{\prime} m^{-\frac{\log _{2} \frac{1}{\rho}}{\log _{2} A}}
\end{aligned}
$$

which is a power law. This rules out the "risk sensitive" sense of stability in which we require $f$ to decrease exponentially. In the context of theorem 4.3, this also implies that there is an $\eta$ beyond which all moments must be infinite! If any unstable process is controlled over a discrete memoryless channel with no zero-error capacity, then the resulting state can have at best a power-law bound ${ }^{24}$ (Pareto distribution) on its tail.

Similarly, if we were to impose a hard limit on actuator effort $(|U| \leq \mathcal{U}$ for some $\mathcal{U}>0)$, then we know that the only way to maintain stability is to also have a hard limit on how big the state $X$ can get. From that, Theorem 4.5 immediately gives a fundamental requirement for feedback zero-error capacity $\geq \log _{2} A$ since $g(d)=0$ for sufficiently large $d$.

Thus for DMCS and a given $A$, we either can achieve at best a power-law tail, or it is possible to hold the state inside a finite box. Nothing in between can happen with a DMC.

\subsubsection{The AWGN case with an average input power constraint}

The tight relationship between control and communication established in theorem 4.5 allows us to construct sequential codes for noisy channels with noiseless feedback if we know how to stabilize linear plants over such channels. Consider the problem of stabilizing an unstable plant driven by finite variance driving noise over an AWGN channel. A linear observer and controller strategy achieve mean-square stability for such systems since the problem fits into the standard LQG framework. [33] In particular, it is known that the system can be stabilized in closed loop by linear controllers and observers if $\log _{2} A<C$ where $C=\frac{1}{2} \log _{2}\left(1+\frac{P}{\sigma^{2}}\right)$ is the Shannon capacity of the AWGN channel with an input power constraint $P$ and channel noise variance $\sigma^{2}$.

Since the $W_{t}$ that we generate are clearly bounded variance, the immediate application of theorem 4.3 tells us that we have an anytime reliability of at least $2 \log _{2} A \geq 2 R$ which can be made as close to $2 C$ as we would like. However, by looking more closely at the actual tail probabilities achieved by the linear observer/controller strategy, we get a natural anytime generalization of Schalkwijk and Kailath's scheme[25] for communicating over the power constrained additive white Gaussian noise channel with noiseless feedback that achieves doubly exponential reliability.

Theorem 4.6 It is possible to communicate bits reliably across a discrete-time power constrained $A W G N$ channel with noiseless feedback at all rates $R<\frac{1}{2} \log _{2}\left(1+\frac{P}{\sigma^{2}}\right)$ while achieving a $g$-anytime reliability of at least

$$
g(d)=2 e^{-K\left(4^{R d}-O\left(2^{R d}\right)\right)}
$$

for some constant $K$ that depends only on the rate $R$, power constraint $P$, and channel noise power $\sigma^{2}$.

\footnotetext{
${ }^{24}$ This is very much related to how sequential decoding must have computational effort distributions with at best a Pareto distribution.[12] In both cases, the result follows from the interaction of two exponentials. The difference is that the computational effort distributions assumed a particular structure on the decoding algorithm while the bound here is fundamental to the stabilization problem no matter what sort of observers or controllers we choose to implement.
} 
Proof: To avoid having to drag $\sigma^{2}$ around, just normalize units so as to consider power constraint $P^{\prime}=\frac{P}{\sigma^{2}}$ and a channel with i.i.d. unit variance noise $N_{t}$. The observer/encoder we use is a linear map

$$
a_{t}=\beta X_{t}
$$

so the channel output $B_{t}=\beta X_{t}+N_{t}$. For the controller, we will assume that it too is linear

$$
U_{t}=-A \phi B_{t}
$$

giving us the closed-loop system:

$$
X_{t+1}=A(1-\beta \phi) X_{t}+W_{t}-A \phi N_{t}
$$

where the $\beta, \phi$ are constants to be chosen. For the closed-loop system to be stable, we must ensure that

$$
0<A(1-\beta \phi)<1
$$

This implies that $\beta \phi \in\left(1-\frac{1}{A}, 1\right)$. Assuming (16) holds and temporarily setting the $W_{t}=0$ for analysis, we see that the closed-loop $X_{t}$ is Gaussian with a growing variance asymptotically tending to

$$
\sigma_{x}^{2}=\frac{A^{2} \phi^{2}}{1-A^{2}(1-\beta \phi)^{2}}
$$

The channel input power satisfies:

$$
E\left[a_{t}^{2}\right] \leq \frac{A^{2}(\beta \phi)^{2}}{1-A^{2}(1-\beta \phi)^{2}}
$$

Because we have some slack in the rate $R<\log _{2} A<\frac{1}{2} \log _{2}\left(1+P^{\prime}\right)$, we know that $A^{2}<1+P^{\prime}$. So, set $P^{\prime \prime}=A^{2}-1<P^{\prime}$. By substituting, we get

$$
E\left[a_{t}^{2}\right] \leq \frac{\left(P^{\prime \prime}+1\right)(\beta \phi)^{2}}{1-\left(P^{\prime \prime}+1\right)(1-\beta \phi)^{2}}
$$

By setting $\beta \phi=\frac{P^{\prime \prime}}{P^{\prime \prime}+1}$, we immediately get that the left hand side of $(18)$ is identically $P^{\prime \prime}$ as we wanted. All that remains is to check the stability condition (16) to see that:

$$
\begin{aligned}
A(1-\beta \phi) & =\frac{A}{P^{\prime \prime}+1} \\
& =\frac{\sqrt{P^{\prime \prime}+1}}{P^{\prime \prime}+1} \\
& =\frac{1}{\sqrt{P^{\prime \prime}+1}} \\
& <1
\end{aligned}
$$

So the closed loop system is stable and the channel noise alone results in an average input power of at most $P^{\prime \prime}<P^{\prime}$.

At this point, we could choose to optimize our choice of $\beta$ and $\phi$ to get the best tradeoff point. For simplicity, just set $\beta=1$ and $\phi=\frac{P^{\prime \prime}}{P^{\prime \prime}+1}$. In that case, we know that $\sigma_{x}^{2}=P^{\prime \prime}$. 
Now consider the impact of the $W_{t}$ alone on the closed-loop control system. These are going through a stable system and so we know by expanding the recursion (15) that:

$$
\begin{aligned}
\left|X_{t}^{w}\right| & \leq \sum_{i=0}^{\infty}(A(1-\beta \phi))^{i} \frac{\Omega}{2} \\
& =\sum_{i=0}^{\infty}\left(\frac{1}{\sqrt{P^{\prime \prime}+1}}\right)^{i} \frac{\Omega}{2} \\
& =\frac{\Omega}{2\left(1-\frac{1}{\sqrt{P^{\prime \prime}+1}}\right)} \\
& =\frac{\Omega \sqrt{P^{\prime \prime}+1}}{2\left(\sqrt{P^{\prime \prime}+1}-1\right)}
\end{aligned}
$$

which is a constant that can be made as small as we like by choice of $\Omega$. Assume that the data stream $S$ to be transmitted is independent of the channel noise $N$. Then, the total average input power is bounded by:

$$
\begin{aligned}
\sigma_{x}^{2}+\left|X_{t}^{w}\right|^{2} & \leq P^{\prime \prime}+\left(\frac{\Omega \sqrt{P^{\prime \prime}+1}}{2\left(\sqrt{P^{\prime \prime}+1}-1\right)}\right)^{2} \\
& \leq P^{\prime \prime}+\Omega^{2} \frac{P^{\prime \prime}+1}{4\left(P^{\prime \prime}+2\left(1-\sqrt{P^{\prime \prime}+1}\right)\right)}
\end{aligned}
$$

Since $P^{\prime \prime}<P^{\prime}$, we can choose an $\Omega$ small enough so that we fit inside the average power constraint no matter what disturbance we are subject to.

All that remains is to see what $f(m)$ this control system meets for such arbitrary, but bounded, disturbances. $X_{t}$ is asymptotically the sum of a Gaussian with zero mean and variance $P^{\prime \prime}$ together with the closed-loop impact of the disturbance $X^{w}(t)$. Since the total impact of the disturbance part is bounded, we know:

$$
\begin{aligned}
\mathcal{P}\left(\left|X_{t}\right|>m\right) & \leq \mathcal{P}\left(\left|N_{\sigma_{x}^{2}}\right|>m-\frac{\Omega \sqrt{P^{\prime \prime}+1}}{2\left(\sqrt{P^{\prime \prime}+1}-1\right)}\right) \\
& =\mathcal{P}\left(|N|>\frac{1}{\sqrt{P^{\prime \prime}}}\left(m-\frac{\Omega \sqrt{P^{\prime \prime}+1}}{2\left(\sqrt{P^{\prime \prime}+1}-1\right)}\right)\right) \\
& \leq 2 e^{-\frac{1}{2 P^{\prime \prime}}\left(m-\frac{\Omega \sqrt{P^{\prime \prime}+1}}{2\left(\sqrt{P^{\prime \prime}+1}-1\right)}\right)^{2}}
\end{aligned}
$$

Ignoring the details of the constants, we have an $f(m)=2 e^{-K_{1}\left(m-K_{2}\right)^{2}}=2 e^{-K_{3}\left(m^{2}-2 K_{2} m\right)}$. Applying theorem 4.5 immediately gives (12) since $A^{d}>2^{R d}$.

Since we get double exponential convergence, it is faster than any exponential and hence

$$
C_{\text {any }}(\alpha)=\frac{1}{2} \log _{2}\left(1+\frac{P}{\sigma^{2}}\right)
$$

for all $\alpha>0$ on the AWGN channel. If the additive channel noise were not Gaussian, but had bounded support with the same variance, then this proof immediately tells us that the zero-error capacity of such a bounded noise channel with feedback satisfies: $C_{0} \geq \frac{1}{2} \log _{2}\left(1+\frac{P}{\sigma^{2}}\right)$. 
In the Gaussian case, it is not immediately clear whether there are ideas analogous to those in [13] that can be used to further boost the $g$-anytime reliability beyond double exponential. It is clear that if it were possible, it would require nonlinear control strategies.

The AWGN case is merely one example. Theorem 4.5 gives us a way to compute the anytime capacity for channels with feedback by allowing us to use cases where the optimal control behavior is easy to see. Looking at the finiteness of the closed-loop state moments tells us what anytime reliability is being achieved. An example of this is the binary erasure channel discussed in [18] and [19]. In addition, theorem 4.5 gives us the ability to mix and match communication and control tools to study a problem. This is exploited in $[21,22]$ to understand the feedback anytime capacity of constrained packet erasure channels and the power constrained AWGN+erasure channel. It is also exploited in [30] to lower bound the anytime reliability achieved by a particular code for the BSC with feedback.

\section{The sufficiency of anytime capacity}

\subsection{Notation specific to this section}

$\bar{U}_{t}$ The scalar virtual control applied to the virtual controlled process $\bar{X}_{t+1}$ in (19).

$\hat{U}_{i}(t)$ At time $t$, the best estimate at the controller for the virtual control $\bar{U}_{i}$ based on the most current message estimates from the anytime decoder.

$\bar{X}_{t}$ The virtual controlled process internal to the observer. This process is used to encode the state evolution causally into bits. Defined in (19).

$X_{t}^{\bar{U}}$ The counterpart to $\tilde{X}_{t}$ except driven only by the virtual controls $\bar{U}_{t}$. Defined in $(20)$.

$\hat{X}_{t+1}(t)$ The best estimate of $-\check{X}_{t+1}$ (the disturbance driven open-loop plant) available to the controller at the end of time $t$, given by (21).

$\Gamma$ The bound on the size of the observation noise on the state $X_{t}$.

$\Delta$ The size of the box that the observer attempts to keep the virtual process within.

\subsection{Overview}

When characterizing a noisy channel for control, the choice of information pattern[38] can be critical.[33] We first establish the result in cases where there is some noiseless feedback path from the channel outputs back to the observer. At first, we assume that the observer has access to all the past and current control signals $U_{t}=u_{t}$ and states $X_{t}=x_{t}$. Then, in section 5.6, the impact of taking time in blocks of size $n$ and only acting on the slower time-scale is examined. Finally, section 5.7 gives a model for boundedly noisy observations or quantization and shows that such observation noise can be tolerated at the observer.

In order to show sufficiency, we assume that we have access to a uniform anytime code that works with noiseless feedback from the channel output available at the bit encoder. This communication system works in terms of bits and generates appropriate channel inputs. We need to design an observer/controller pair that deals with the analog plant and communicates across the channel by 
using the anytime communication system. This will allow us to prove our first general theorem, dealing with the problem illustrated in figure 2 .

Theorem 5.1 For a given noisy channel, if there exists an anytime encoder/decoder pair with access to noiseless feedback that achieves $C_{g-a n y}(g) \geq \log _{2} A$, then it is possible to control an unstable scalar plant with parameter $A$ that is driven by bounded driving noise through the noisy channel by using an observer that has access to the noisy channel outputs. Furthermore, there exists a constant $K$ so that $\mathcal{P}\left(\left|X_{t}\right|>m\right) \leq g\left(K+\log _{A} m\right)$.

To prove this theorem, we give constructions for the observer and controller.

\subsection{Observer}

Since the observer has access to the channel outputs, it can run a copy of the controller and so has access to the control signals as well. The observer is constructed to keep the state uncertainty inside a box of size $\Delta$ by using bits at the rate $R$. It does this by controlling a virtual process $\bar{X}_{t}$ governed by:

$$
\bar{X}_{t+1}=A \bar{X}_{t}+W_{t}+\bar{U}_{t}
$$

This gives rise to a virtual counterpart of $\tilde{X}_{t}$

$$
X_{t+1}^{\bar{U}}=A X_{t}^{\bar{U}}+\bar{U}_{t}
$$

which satisfies the relationship $\bar{X}_{t}=\check{X}_{t}+X_{t}^{\bar{U}}$. Because $\bar{X}_{t}$ will be kept within a box, we will know that $-X_{t}^{\bar{U}}$ is close to $\check{X}_{t}$. The controller will pick controls designed to keep $\tilde{X}_{t}$ close to $X_{t}^{\bar{U}}$.

The goal of the virtual process is to pretend that we have a noiseless rate $R$ channel to the controller in the manner of example 3.1. To simulate $\bar{X}_{t+1}$ the observer computes $W_{t}=X_{t+1}-$ $A X_{t}-U_{t}$. Because of the rate constraint, the virtual control $\bar{U}_{t}$ takes on one of $2^{\lfloor R(t+1)\rfloor-\lfloor R t\rfloor}$ values. For simplicity of exposition, we will ignore the integer effects and consider it to be one of $2^{R}$ values ${ }^{25}$. We proceed by induction. Suppose that $\bar{X}_{t}$ is known to lie within $\left[-\frac{\Delta}{2}, \frac{\Delta}{2}\right]$. Then $A \bar{X}_{t}$ will lie within $\left[-\frac{A \Delta}{2}, \frac{A \Delta}{2}\right]$. By choosing $2^{R}$ control values uniformly spaced within that interval, we can guarantee that $A X_{t}+\bar{U}_{t}$ will lie within $\left[-\frac{A \Delta}{2^{R+1}}, \frac{A \Delta}{2^{R+1}}\right]$. Finally, the state will be disturbed and $\bar{X}_{t+1}$ will be known to lie within $\left[-\frac{A \Delta}{2^{R+1}}-\frac{\Omega}{2}, \frac{A \Delta}{2^{R+1}}+\frac{\Omega}{2}\right]$.

To get a handle on the minimum $\Delta$ required as a function of $R$, we now solve for the steady state where

$$
\frac{A \Delta}{2^{R}}+\Omega \leq \Delta
$$

reaches equality. This occurs ${ }^{26}$ when $\Delta=\frac{\Omega}{1-A 2^{-R}}$ for every case where $R>\log _{2} A$. If $R<\log _{2} A$, the state $\bar{X}$ can grow without bound.

It is clear that the virtual controls $\bar{U}_{t}$ can be encoded causally using $R$ bits per unit time. These bits are sent to the anytime encoder for transport over the noisy channel. ${ }^{27}$

\footnotetext{
${ }^{25}$ For the details of how to deal with fractional $R$, please see the causal source code discussion in [19].

${ }^{26}$ In reality, our uncertainty approaches this from below since we start at the known initial condition 0

${ }^{27}$ Because the actual controls are being subtracted out, the output of the observer is completely independent of the channel noise.
} 


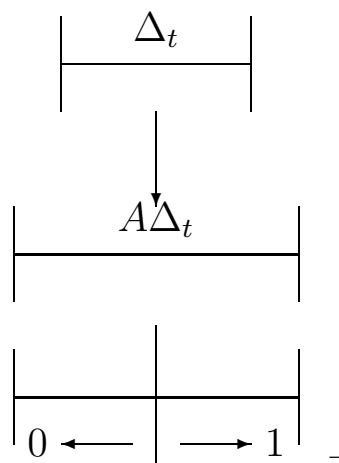

Window known to contain $\bar{X}_{t}$

will grow by factor of $A>1$ due to the dynamics

By sending $R$ bits, we cut the window by a factor of $2^{-R}$

Encode which virtual control $\bar{U}_{t}$ to apply

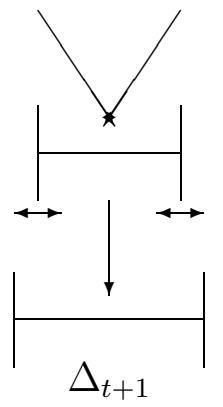

grows by $\frac{\Omega}{2}$ on each side

giving a new window for $\bar{X}_{t+1}$

Figure 6: Virtual controller illustrated for $\mathrm{R}=1$. How $\bar{X}$ evolves.

\subsection{Controller}

We assume that the encoder has memory of the past controls applied. The controller uses the regularly updated bit estimates from the anytime decoder to apply a control so as to attempt to make the true state $X_{t}$ stay close to the virtual state $\bar{X}_{t}$. It does this by having a pair of internal models as shown in figure 7 .

The first models the unstable system driven only by the actual controls. This is referred to as $\tilde{X}_{t}$ in (3) above. The second is its best estimate $\hat{X}_{t}$, based on the current bit estimates from the anytime decoder, of where the unstable system should be driven only by the virtual controls $\bar{U}_{t}$. Of course, we do not have the exact virtual controls, only our best estimates $\hat{U}_{1}^{i}(t)$ for them.

$$
\hat{X}_{t+1}(t)=\sum_{i=0}^{t} A^{i} \hat{U}_{t-i}(t)
$$

Notice that this is not given in recursive form since all of the past estimates for the virtual controls are subject to re-estimation at the current $t$. We then apply a control $U_{t}$ designed to make $\tilde{X}_{t+1}=$ $\hat{X}_{t+1}(t)$.

$$
U_{t}=\hat{X}_{t+1}(t)-A \tilde{X}_{t}
$$

\subsection{Evaluating stability}

Proof of theorem 5.1: With controls given by (22), the true state $X_{t}$ can be written as:

$$
\begin{aligned}
X_{t} & =\check{X}_{t}+\tilde{X}_{t}=\check{X}_{t}+\hat{X}_{t}(t-1) \\
& =\sum_{i=0}^{t-1} A^{i}\left(W_{t-i}+\hat{U}_{t-i}(t-1)\right)
\end{aligned}
$$




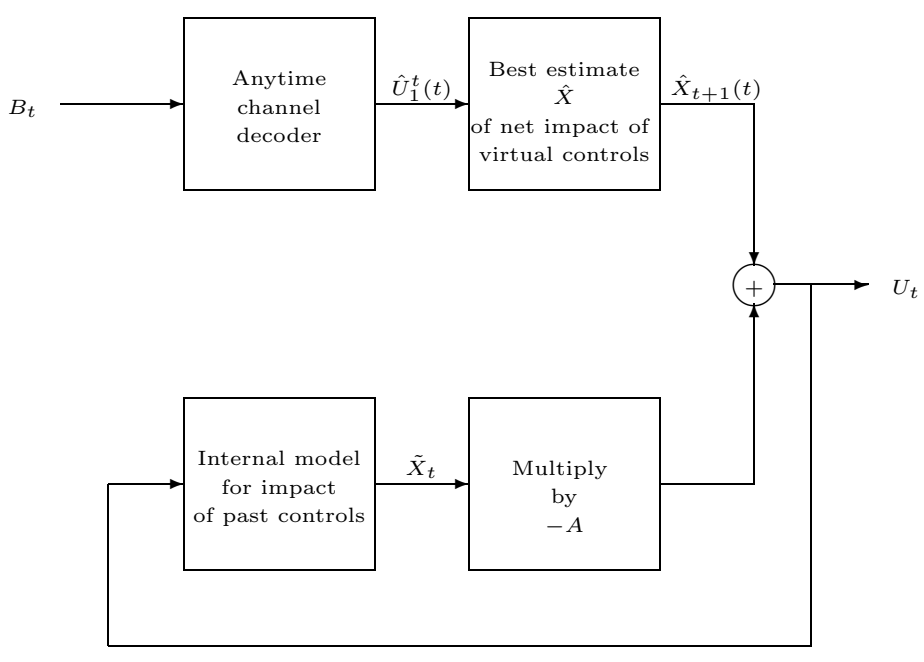

Figure 7: The controller remembers what it did in the past and uses the anytime decoder to get an updated sense of where the observer wants it to go. It then applies a control designed to correct for any impact of past errors and move the state to be close to the virtual state controlled by the observer.

Notice that the actual state $X_{t}$ differs from the virtual state $\bar{X}_{t}$ only due to errors in virtual control estimation due to channel noise. If there were no errors in the prefix $\hat{U}_{1}^{t-d}$ and arbitrarily bad errors for $\hat{U}_{t-d+1}^{t}$, then we could start at $\bar{X}_{t-d}$ and see how much the errors could have propagated since then:

$$
X_{t}=A^{d} \bar{X}_{t-d}+\sum_{i=0}^{d-1} A^{i}\left(W_{t-i}+\hat{U}_{t-i}(t-1)\right)
$$

Comparing this with $\bar{X}_{t}$, and noticing that the maximum difference between two virtual controls is $2 A \Delta$ gives us:

$$
\begin{aligned}
\left|X_{t}-\bar{X}_{t}\right| & =\left|\sum_{i=0}^{d-1} A^{i}\left(\bar{U}_{t-i}-\hat{U}_{t-i}(t-1)\right)\right| \\
& \leq \sum_{i=0}^{d-1} A^{i}\left|\bar{U}_{t-i}-\hat{U}_{t-i}(t-1)\right| \\
& \leq \sum_{i=0}^{d-1} A^{i+1} 2 \Delta \\
& <2 \Delta A^{d} \sum_{i=0}^{\infty} A^{-i} \\
& =A^{d} \frac{2 \Delta}{1-A^{-1}}
\end{aligned}
$$

Since $\left|\bar{X}_{t}\right| \leq \frac{\Delta}{2}$, if we know that there were no errors in the prefix of estimated virtual controls up through $d$ time steps back, then

$$
\left|X_{t}\right|<A^{d} \frac{3 \Delta}{1-A^{-1}}
$$


In other words, for $\left|X_{t}\right|$ to be larger than $A^{d} \frac{3 \Delta}{1-A^{-1}}$ it requires for there to be an error in decoding the messages from $d$ time steps ago or more. (23) immediately gives us a way to connect the bound on the probability of prefix error given by the anytime reliability to a bound on the probability of large deviation in $X_{t}$.

$$
\begin{aligned}
\mathcal{P}\left(\left|X_{t}\right| \geq m\right) & =\mathcal{P}\left(\left|X_{t}\right| \geq A^{\frac{\log _{2} m}{\log _{2} A}} A^{\frac{\log _{2}\left(1-A^{-1}\right)-\log _{2}(3 \Delta)}{\log _{2} A}}\left(\frac{3 \Delta}{1-A^{-1}}\right)\right) \\
& \leq \mathcal{P}\left(\left|X_{t}\right| \geq A^{\left[\frac{\log _{2} m+\log _{2}\left(1-A^{-1}\right)-\log _{2}(3 \Delta)}{\log _{2} A}\right.}\left(\frac{3 \Delta}{1-A^{-1}}\right)\right) \\
& \leq g\left(\left[\frac{\log _{2} m+\log _{2}\left(1-A^{-1}\right)-\log _{2}(3 \Delta)}{\log _{2} A}\right\rceil\right) \\
& \leq g\left(K^{\prime \prime}+\frac{\log _{2} m}{\log _{2} A}\right)
\end{aligned}
$$

where $g$ bounds the probability of error for the $g$-anytime code and $K^{\prime \prime}$ is some constant.

Specializing to the case of $\alpha$-anytime capacity, we see that we get:

$$
\begin{aligned}
\mathcal{P}\left(\left|X_{t}\right| \geq m\right) & \leq K^{\prime \prime \prime} 2^{-\alpha \frac{\log _{2} m}{\log _{2} A}} \\
& =K^{\prime \prime \prime} m^{-\frac{\alpha}{\log _{2} A}}
\end{aligned}
$$

which gives a power-law bound on the tail. From this we see that if we want to hold the $\eta$-th moment bounded,

$$
\begin{aligned}
E\left[\left|X_{t}\right|^{\eta}\right] & =\int_{0}^{\infty} \mathcal{P}\left(\left|X_{t}\right|^{\eta} \geq m\right) d m \\
& =\int_{0}^{\infty} \mathcal{P}\left(\left|X_{t}\right| \geq m^{\frac{1}{\eta}}\right) d m \\
& \leq 1+K^{\prime \prime \prime} \int_{1}^{\infty} m^{-\frac{\alpha}{\eta \log _{2} A}} d m
\end{aligned}
$$

As long as $\alpha>\eta \log _{2} A$, the integral above converges and hence the controlled process has a bounded $\eta$-moment. This result is summarized in the following theorem:

Theorem 5.2 It is possible to control an unstable scalar process driven by a bounded disturbance over a noisy channel so that the $\eta$-moment of $X_{t}$ stays finite for all time if the channel with feedback has $C_{\text {any }}(\alpha)>\log _{2} A$ for some $\alpha>\eta \log _{2} A$ and the observer is allowed to observe the noisy channel outputs and the state exactly.

Aside from the usual gap between $>$ and $\geq$, this shows that the necessity condition in theorem 4.3 is tight. Also, notice that we are making no assumptions on the disturbance process except for its boundedness. All the sufficiency theorems here automatically cover the case of stochastic disturbances having any sort of memory structure as long as they remain bounded in support. 


\subsection{Time in blocks and delayed observations}

Suppose we group time into blocks of size $n$ and restrict our observer to only encode the value of $X_{t}$ at times that are integer multiples of $n$. In such cases, we can also ask that the control only take an action ${ }^{28}$ immediately before the encoder will sample the state and the effective system dynamics change to

$$
X_{n(k+1)}=A^{n} X_{n k}+U_{n(k+1)-1}+W_{k}^{\prime}
$$

where $W_{k}^{\prime}=\sum_{j=0}^{n-1} A^{n-1-j} W_{n k+j}$. Observe that $\left|W_{k}^{\prime}\right|$ is known to be bounded within an interval of size $\Omega^{\prime}<A^{n} \frac{\Omega}{A-1}$. Essentially, everything has just scaled up by a factor of $A^{n}$. Thus all the results above will continue to hold above for a system described by (24) at times which are integer multiples of $n$. The rate must be larger than $\log _{2} A^{n}=n \log _{2} A$ bits per $n$ time steps which translates to $\log _{2} A$ bits per time step. The anytime reliability $\alpha>\eta \log _{2} A^{n}=n\left(\eta \log _{2} A\right)$ per for delay measured in units of $n$ time-steps which translates into $\alpha>\eta \log _{2} A$ for delay measured in unit time steps. This is the same as it was for the system described by (1).

The only question that remains is what happens to the state at times within a block of size $n$ since no controls are being applied while the state continues to grow on its own. At such times, the state has just grown by a factor of at most $A^{n}$ with an additive term of at most $A^{n} \frac{\Omega}{A-1}$.

$$
\begin{aligned}
E\left[\left(A^{n}\left(X_{n k}+\frac{\Omega}{A-1}\right)\right)^{\eta}\right] & =A^{\eta n} E\left[\left(X_{n k}+\frac{\Omega}{A-1}\right)^{\eta}\right] \\
& \leq A^{\eta n} E\left[\left(2 \max \left(\left|X_{n k}\right|, \frac{\Omega}{A-1}\right)\right)^{\eta}\right] \\
& =A^{\eta n} 2^{\eta} \int_{0}^{\infty} \mathcal{P}\left(\max \left(\left|X_{n k}\right|^{\eta},\left(\frac{\Omega}{A-1}\right)^{\eta}\right) \geq \tau\right) d \tau \\
& =A^{\eta n} 2^{\eta}\left(\left(\frac{\Omega}{A-1}\right)^{\eta}+\int_{\left(\frac{\Omega}{A-1}\right)^{\eta}}^{\infty} \mathcal{P}\left(\left|X_{n k}\right|^{\eta} \geq \tau\right) d \tau\right) \\
& <A^{\eta n} 2^{\eta}\left(\left(\frac{\Omega}{A-1}\right)^{\eta}+\int_{0}^{\infty} \mathcal{P}\left(\left|X_{n k}\right|^{\eta} \geq \tau\right) d \tau\right) \\
& =A^{\eta n} 2^{\eta}\left(\left(\frac{\Omega}{A-1}\right)^{\eta}+E\left[\left|X_{n k}\right|^{\eta}\right]\right)
\end{aligned}
$$

which is finite since the original is finite. Thus we know that Theorem 5.2 and by similar arguments theorem 4.3 continue to hold even if we only get access to the system at timesteps that are integer multiples of some $n$. We will use this when considering noisy observations in section 5.7 and again in the context of vector-valued states in section 8 .

\subsection{Noisy or quantized observations}

In the proof of Theorem 5.2, we used exact knowledge of the state $X_{t}$ in the construction of the observer in section 5.3. Suppose instead that the encoder only has access to $X_{n o i s y}(t)=X_{t}+N_{t}$ where $N_{t}$ is known to be within a bound $\left(\frac{-\Gamma}{2}, \frac{+\Gamma}{2}\right)$. For example, this models situations where the input to the encoder has already been quantized to some resolution. ${ }^{29}$

\footnotetext{
${ }^{28}$ The controller can take "no action" by setting the control signal to zero.

${ }^{29}$ The quantization is assumed to be to some coarseness, but we assume that the dynamic range of the quantization is still infinite. Section 4.4 tells us that a finite dynamic range on the quantization will impose the requirement of zero-error capacity on the link.
} 


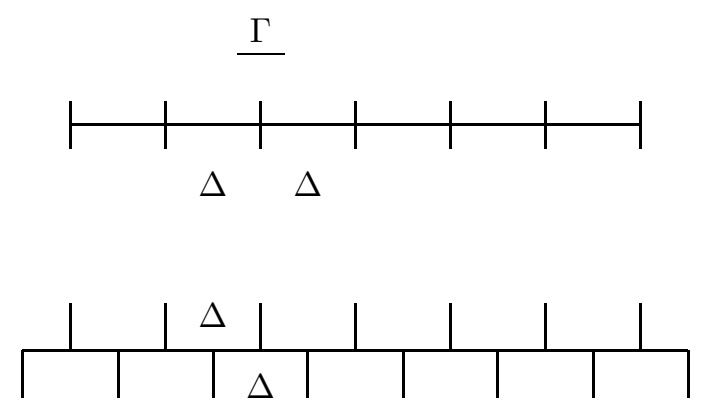

Figure 8: When there is noise in the observations, no strict partition of the line can adequately capture the uncertainty since it can straddle the boundary of two regions. By doubling the number of bins, we can be certain that a bin exists that completely contains the uncertainty from the noisy observation. This doubling adds an overhead of 1 bit.

We need to ensure that the virtual state $\bar{X}$ is within an interval of size $\Delta$. To do this, we will just choose a large enough $\Delta>2 \Gamma$ so that if $X_{n o i s y}(t)$ is used to pick which interval we are in, we can guarantee that the precise value $X_{t}$ would have given us the same interval. As figure 8 illustrates, this is not quite enough since the intervals used in section 5.3 are partitions of the real line. Meanwhile, each observation of $X_{\text {noisy }}(t)$ gives rise to an uncertainty window for $X_{t} \in\left(X_{\text {noisy }}(t)-\frac{\Gamma}{2}, X_{\text {noisy }}(t)+\frac{\Gamma}{2}\right)$ that might straddle a boundary of the partition. ${ }^{30}$ To have the intervals always able to hold the entire uncertain window, we can double the number of intervals and have them overlap by half. Doubling the number of intervals increases the data rate by at most an additional bit. To amortize this additional bit, we use section 5.6 and look at time in blocks of size $n$. Then, the required rate for achievability with blocked time will be $R>1+\log _{2} A^{n}$ bits per $n$ time-steps or $R>\frac{1}{n}+\log _{2} A$ bits per time step. By choosing $n$ large enough, all we require is $R>\log _{2} A$. Since the controls are known to us perfectly from the perfect channel feedback, they cause no new concern. Thus, we get the following corollary to Theorem 5.2:

Corollary 5.1 It is possible to control an unstable scalar process driven by a bounded disturbance over a noisy channel so that the $\eta$-moment of $X_{t}$ stays finite for all time if the channel with noiseless feedback has $C_{\text {any }}(\alpha)>\log _{2} A$ for some $\alpha>\eta \log _{2} A$ and the observer is only allowed to observe the noisy channel outputs exactly and has a boundedly noisy view of the state.

\section{$6 \quad$ Relaxing feedback}

In this section, we relax the assumption that the observer can observe the outputs of the noisy channel directly. This change of information pattern has the potential to make the problem more difficult. We restrict the information pattern in stages. First, we consider the problem of figure 9 in which the observer can see the controls but not the channel outputs. Then, we consider the problem of figure 12 that restricts the observer to only see the states $X_{t}$. We divide this section based on the approach rather than the problem.

In subsection 6.1, the solutions are based on using anytime codes without feedback. These give rise to sufficient conditions that are not tight with the necessary conditions established in theorem

\footnotetext{
${ }^{30}$ This will not arise for statically quantized states since those will have fixed boundaries. In that case, nothing needs to be done except ensuring that the partitions we use respect those boundaries.
} 


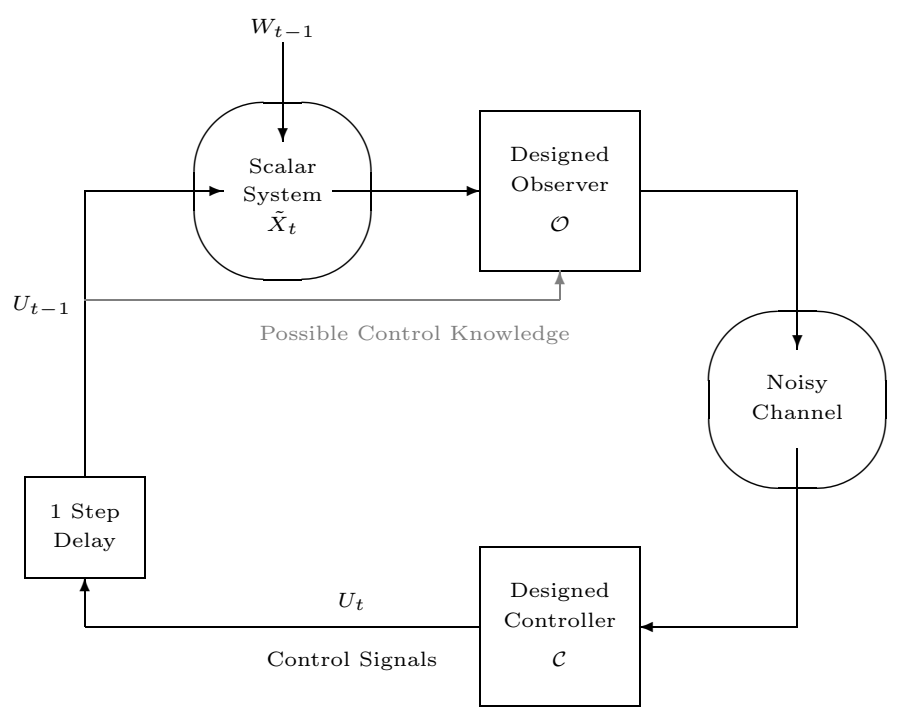

Figure 9: Control over a noisy communication channel without explicit feedback of channel outputs.

4.3. The main result is theorem 6.3 - a random construction that shows it is possible, in the case of DMCs, to have relatively memoryless time-varying observers and still achieve stability in many cases. All the complexity can in principle be shifted to the controller side.

In subsection 6.2, the solutions are based on communicating the channel outputs back to the observer through either the control signals or by making the plant itself "dance" in a stable way that communicates limited information noiselessly with no delay. Such solutions give rise to tight sufficient conditions. These are not as constructive, but serve to establish the fundamental connection between stabilization and communication with noiseless feedback.

\subsection{Using anytime codes without feedback}

In this subsection, we first show that we can get a more restrictive sufficient condition for stabilization by using anytime codes without feedback. This result is robust to noise on the control signals. Then we show how to eliminate all access to the control signals by the encoder and end with a time-varying random quantization approach that works over any DMC.

\subsubsection{Have noisy access to the control signals}

The only place where we used the noiseless access to the channel outputs at the observer was for the operation of the embedded anytime encoder. Without that access, we can use an anytime encoder for the channel without feedback. If the conditions for theorems 5.2 and 5.1 are met using anytime encoder/decoder pairs without access to feedback, then they continue to hold as long as the observer is allowed access to the control signals.

Noisefree access to the control signals was fine in the case of corollary 5.1 since the control signals could be calculated from the perfect channel feedback. Without such perfect feedback, it is more realistic to consider only noisy access to the control signals. We further observe that exact knowledge of the actual applied controls in section 5.3 is only used to calculate $W_{t}$ from the observed $X_{t+1}, X_{t-1}, U_{t}$. As such, any bounded observation noise on the control signals $U_{t}$ just translates into 
an effectively larger $\Gamma$ on the noisy state observations. Since any finite $\Gamma$ can be dealt with, we have:

Theorem 6.1 It is possible to control an unstable scalar process driven by a bounded disturbance over a noisy channel so that the $\eta$-moment of $X_{t}$ stays finite for all time if the channel without feedback has $C_{\text {any }}(\alpha)>\log _{2} A$ for some $\alpha>\eta \log _{2} A$ and the observer is allowed noisy access to the control signals and the state process as long as the noise on both is bounded.

Without noiseless feedback the anytime capacity will tend to be considerably lower ${ }^{31}$ for a given $\alpha$, and so there will be a gap between the necessary condition established in theorem 4.3 and what we have here in Theorem 6.1. Thus a better channel is required for theorem 6.1 than was needed for theorem 5.2.

\subsubsection{No access to the controls at observer}

Next, we consider the problem of figure 12 that restricts the observer to only see the states $X_{t}$. The challenge is that the observer as given in section 5.3 needs to know the controls in order to remove their effect and focus only on encoding the virtual process $\bar{X}_{t}$.

Theorem 6.2 It is possible to control an unstable scalar process driven by a bounded disturbance over a noisy channel so that the $\eta$-moment of $X_{t}$ stays finite for all time if the channel without feedback has $C_{\text {any }}(\alpha)>\log _{2} A$ for some $\alpha>\eta \log _{2} A$ and the observer is allowed noisy access to the state process as long as the noise is bounded.

Furthermore, this is possible by using an observer consisting of a time-invariant quantizer that samples the state every $n$ time steps and passes the quantization bin index to an anytime encoder.

Proof: We will patch the proof for theorem 6.1 to cover this case. Notice that:

$$
\begin{aligned}
\bar{X}_{t} & =\check{X}_{t}+X_{t}^{\bar{U}} \\
& =X_{t}-\tilde{X}_{t}+X_{t}^{\bar{U}} \\
& =X_{t}-\tilde{X}_{t}+A X_{t-1}^{\bar{U}}+\bar{U}_{t-1}
\end{aligned}
$$

This shows that the only impact of knowing the control signals and the past virtual controls at the observer is to appropriately translate the quantization bins and choose the exact values for the $\bar{U}_{t}$ signals corresponding to those bins. The $R$-bit message sent to the anytime encoder is just the quantization bin index. The sequence of bin indices are then interpreted by the controller to pick the next control signal to apply.

At this point, we can treat the $\left(\tilde{X}_{t}, A X_{t-1}^{\bar{U}}\right)$ as side information that is known perfectly at the intended recipient (the controller) and use lattice binning to eliminate the need for it at the encoder. The idea is simple - instead of having just one window looking at the appropriate neighborhood of $X_{t}$ and covering it with quantization bins as was done in figure 8, we just copy the window periodically in both directions so that it covers the entire real line. The result is illustrated in figure 10.

To account for the lack of precise centering of the bins, we must add only one additional bin. However, we have already doubled the number to account for the bounded observation noise on $X$ so

\footnotetext{
${ }^{31} \mathrm{~A}$ technique due to Pinsker [15] can be used to show that for symmetric DMC channels, the anytime reliability can not exceed the sphere-packing bound at any rate. The anytime reliability with feedback can be substantially higher.[30] Pinsker had claimed otherwise in [15], but he was in error.[24]
} 


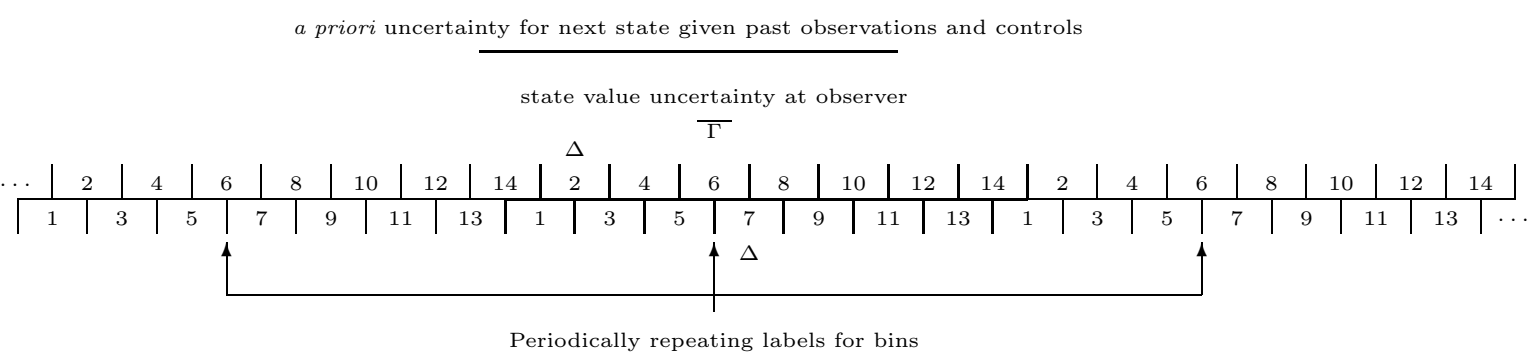

Figure 10: If the observer does not have the controls or a memory of the past virtual controls, it can extend the quantization lattice infinitely in both directions and repeat the bin labels periodically. If the observer had known the controls, it would have centered the lattice to cover the top bar exactly. However, it does not know and so we must add one additional quantization bin at the end so that no matter how the uncertainty ends up being positioned, it never covers two bins bearing the same label.

this adds at most 2 bits of overhead. Once again this can be amortized by looking at time in blocks of $n$ and so does not asymptotically cost any rate.

The controller first has to interpret the best estimates $\hat{M}_{1}^{t}(t)$ for the sent messages so far. It knows the positioning of the first uncertainty interval and can thus interpret the first bin index $\hat{M}_{1}(t)$ and compute the appropriate $\bar{U}_{1}$. Assume that it knows how to interpret everything correctly up to the $i$-th bin index and so has $X_{i}^{\bar{U}}$. It also has a perfect memory of its applied control $U_{i}$ and so can position the $i+1$-th uncertainty interval and interpret the $i+1$-th bin index that it receives. The only way it can make a mistake in the interpretation at the $(i+1)$-th stage is if it has decoded the bin index itself incorrectly from the anytime code.

Thus the rest of the proof goes through unchanged and the controller's behavior will result in a controlled state that will satisfy the bound (23) if there were no errors in the $\hat{M}_{1}^{t-d}$.

Notice that the source-coding part of the observer has become very simple — just a time-invariant scalar quantizer that samples the state every $n$ time units. The source-decoding complexity has been shifted entirely to the controller side.

\subsubsection{Direct random construction of the observer}

Theorem 6.2 applies to general channels for which we have an anytime code that works without feedback. Theorem 4.1 in [23] tells us that random tree codes with tree labels chosen i.i.d. using the appropriate channel input distribution give an anytime reliability of $\alpha=E_{r}(R)$ the random coding error exponent for a DMC as evaluated in base 2. The corresponding anytime decoder is just the maximum-likelihood decoder that picks the most likely path through the tree based on the channel inputs received so far.

The tree structure to the anytime encoder requires the observer to remember all the past indices that it emitted. This seems strange given that if the control system is successful, the plant state will keep returning to the bins in the neighborhood of the origin. The Markov nature of the plant suggests that this memory at the observer is not necessary. It turns out that this indeed is the case.

Theorem 6.3 It is possible to control an unstable scalar process driven by a bounded disturbance over a DMC so that the $\eta$-moment of $X_{t}$ stays finite for all time if the channel without feedback has 
$E_{r}(R)>\eta \log _{2} A$ for some $R>\log _{2} A$ and the observer is allowed noisy access to the state process as long as the noise is bounded.

Furthermore, this is possible by using an observer consisting of a time-varying random quantizer that samples the state every $n$ time steps and uses a random bin index chosen i.i.d. from the $\mathcal{A}^{n}$ according to the distribution that maximizes the random coding error exponent at $R$. The controller must have access to the common randomness used to choose the the random bin indices.

Proof: Consider what happens if we apply theorem 4.1 from [23] for a DMC to generate the feedbackfree anytime code for use in theorem 6.2. The first part of the result follows immediately since the random tree code has anytime reliability $\alpha=E_{r}(R)$.

Every distinct path taken through the set of possible intervals for $\bar{X}_{t}$ corresponds to distinct path taken through the tree code. This holds even for paths through the set that intersect with one another in a particular interval along the way. This is wasteful since it makes the anytime decoder work hard to disambiguate paths that make no difference since conditioned on the applied control sequence, they physically end up in the same place and thus call for the same next control.

By instead randomly and independently assigning symbols from $\mathcal{A}^{n}$ to the intervals themselves through space and time, we can construct a random trellis code. From each interval in the set, conditioned on the actual control signals applied, there are at most $2^{n R}$ possible intervals that we could end up at. Figure 11 shows what such a trellis looks like.

Fix a time $t$. The knowledge of the past control signals allows us to interpret the trellis. An error event at depth $d$ occurs at the decoder when the maximum likelihood path last intersected with the true path $d$ blocks ago. If the current maximum-likelihood path diverged from the true path $d$ blocks ago, we are only going to make a mistake in the calculation of the impact of the last $d$ virtual controls. As such, (23) applies using $A^{n}$ instead of $A$ to account for the blocking of time.

Because all false paths from that point onward are disjoint from the true path, and there are no more than $2^{\text {ndR }}$ such false paths, we can apply Gallager's random block-coding analysis for a block code of rate $R$ and block-length $n d$. This gives a probability of error that is at most $2^{-d n E_{r}(R)}$ conditioned on last agreeing with the true path $d$ blocks ago. Taking a normal union bound over all delays $\geq d$ still results in an exponent of $d n E_{r}(R)=n \alpha$. So, aside from the blocking of time, the previous analysis applies and everything works fine as long as $\alpha>\eta \log _{2} A$.

Although the condition in theorem 6.3 is not tight, the result has several nice features. First, it allows us to easily verify sufficiency for a good channel since $E_{r}(R)$ is easy to calculate. Second, it tells us that structurally we do not need to use very complex observers. The implicit memory in the plant can play the role of the memory that we would otherwise need to implement in an observer. The complexity can be shifted to the decoder, and even that complexity need not be too bad. We believe that sequential decoding could be used at the decoder since it is known to have the same asymptotic performance with respect to delay as the ML decoder.[11]

However, if all we want is for the probability of large deviations in the state to be bounded above by some $f(m)$ that goes to zero with large $m$, then we are fine with any DMC with Shannon capacity larger than $\log _{2} A$ since $E_{r}(R)>0$ for all rates $R<C$. Because in general an exponential decay in the probability of error is the best we can hope for without feedback, we do not bother stating the result analogous to theorem 4.5 .

Corollary 6.1 If the observer is allowed boundedly noisy access to the plant state, and the noisy channel is a DMC with Shannon capacity $C>\log _{2} A$, then there exists an observer/controller pair 


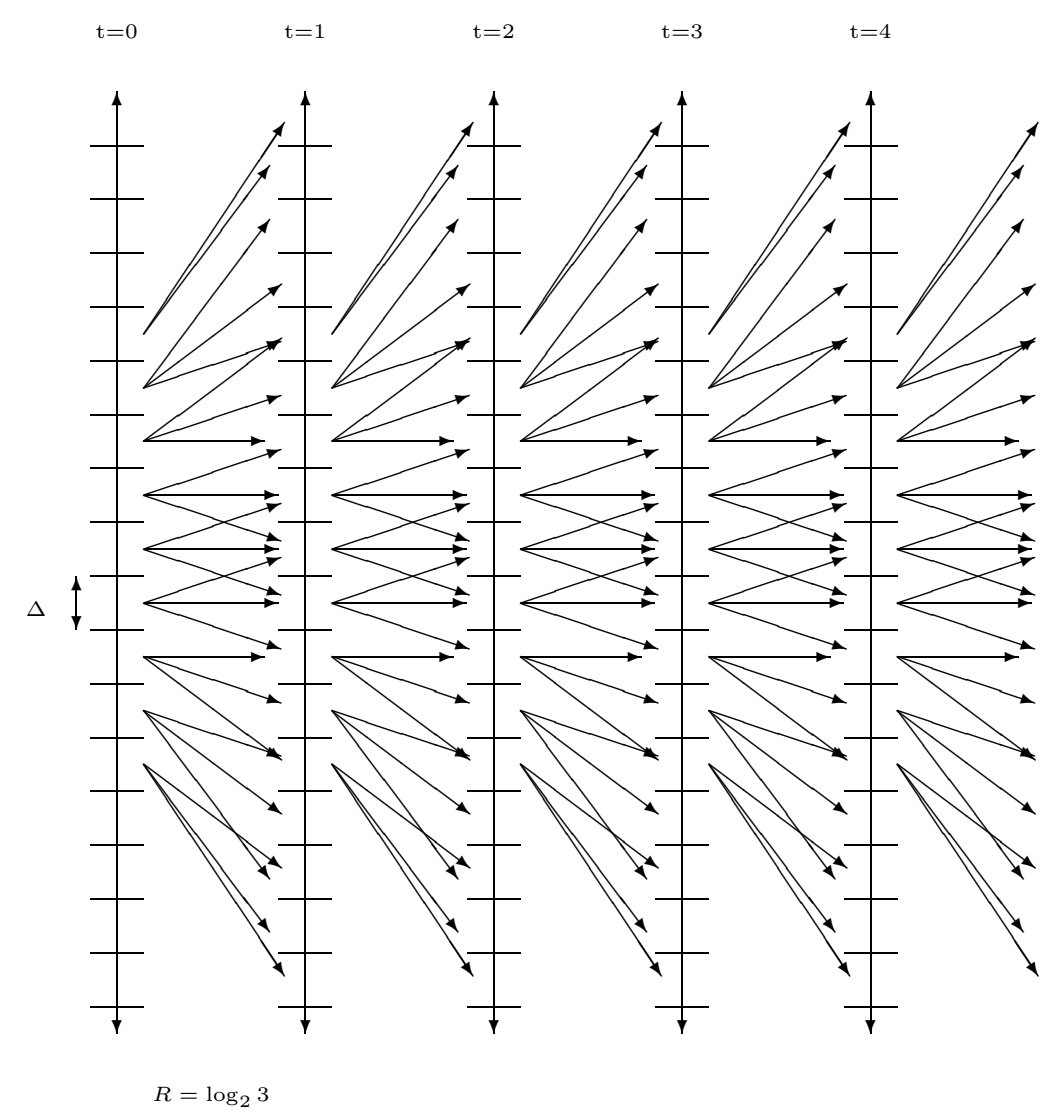

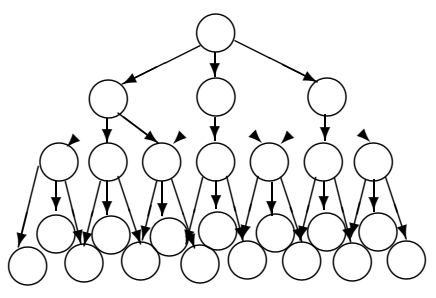

Locally rooted tree-like

structure to the trellis nodes correspond to intervals

where the state might have been

and levels correspond to the time

Random trellis property:

all labels on disjoint paths

are independent of each other

Figure 11: A short segment of the randomly labeled regular trellis from the point of view of the controller that knows the actual control signals applied in the past. The example has $R=\log _{2} 3$ and $A \approx 2.4$ with $\Delta$ large. 
that stabilizes the system in closed loop so that the $\eta$-moment of $X_{t}$ stays finite for all time for some $\eta>0$.

Furthermore, this is possible by using an observer consisting of a time-varying random quantizer that samples the state every $n$ time steps and uses a random bin index chosen i.i.d. from the $\mathcal{A}^{n}$ according to the distribution that maximizes the random coding error exponent at $R$. The controller must have access to the common randomness used to choose the the random bin indices.

\subsection{Communicating the channel outputs back to the observer}

In the previous section, we used anytime codes without feedback to stabilize the system since we did not have access to the channel outputs at the observer. In this section, we will take a different approach. The controller will attempt to explicitly communicate the channel outputs to the observer using the channels available to it. First we consider using the noiseless control signal to embed our feedback information. This motivates the technique we next use to communicate the feedback information by making the plant itself dance in a stable way that tells the observer what the channel output was.

\subsubsection{Using the controls to communicate the channel outputs}

We want to recover the tight condition on the channel from theorem 5.2. The idea is to "cheat" 32 and communicate the channel outputs through the controls. The controller can choose to quantize his real-valued controls to a suitable level and then use the infinite bits remaining in the fractional part to communicate the channel outputs to the observer. The observer can then extract these bits noiselessly and give them to the anytime encoder as noiseless channel feedback. Of course, this fractional part will introduce an added disturbance to the plant. However, the effect of this disturbance is exactly known to the controller and the observer and hence the control can have an additional fractional part that cancels the cumulative effect of past introduced disturbances without interfering with the feedback communication of the channel output.

Theorem 6.4 It is possible to control an unstable scalar process driven by a bounded disturbance over a noisy channel so that the $\eta$-moment of $X_{t}$ stays finite for all time if the channel with feedback has $C_{\text {any }}(\alpha)>\log _{2} A$ for some $\alpha>\eta \log _{2} A$ and the observer is allowed to observe the control signals perfectly.

Proof: Let $b_{t}$ denote the channel output at time $t$. The modified control signal is $U_{t}^{\prime}=Q_{2^{-L}}\left(U_{t}\right)+$ $F\left(b_{t}\right)-A F\left(b_{t-1}\right)$. Here $Q_{2^{-L}}$ quantizes its input to the nearest multiple of $2^{-L}$. $F$ maps the channel output invertibly into the range $\left(-2^{-(L+1)}, 2^{-(L+1)}\right)$. By induction, the observer can remove $A F\left(b_{t-1}\right)$ and will then be able to extract both $Q_{2^{-L}}\left(U_{t}\right)$ and $F\left(b_{t}\right)$ from the binary expansion for $U_{t}^{\prime}$. The net impact on the control of the state will be slight — no more than $2^{-L-1} A$ in either direction. The

\footnotetext{
${ }^{32}$ We call this "cheating" since it violates the spirit of the requirement against access to the channel outputs. However, it is important that we establish this result because it points out the need for a serious future study where we explicitly model communication constraints back from the controller to the observer. A more realistic model for the problem will have a sensor observing the plant connected via a communication channel to the controller. The controller is then connected to an actuator through another communication channel. The actuator finally acts upon the plant itself. With no complexity constraints, this reduces to the case studied here with the controller merely playing the role of a relay bridging together two communication channels. The relay anytime reliability will become the relevant quantity to study.
} 


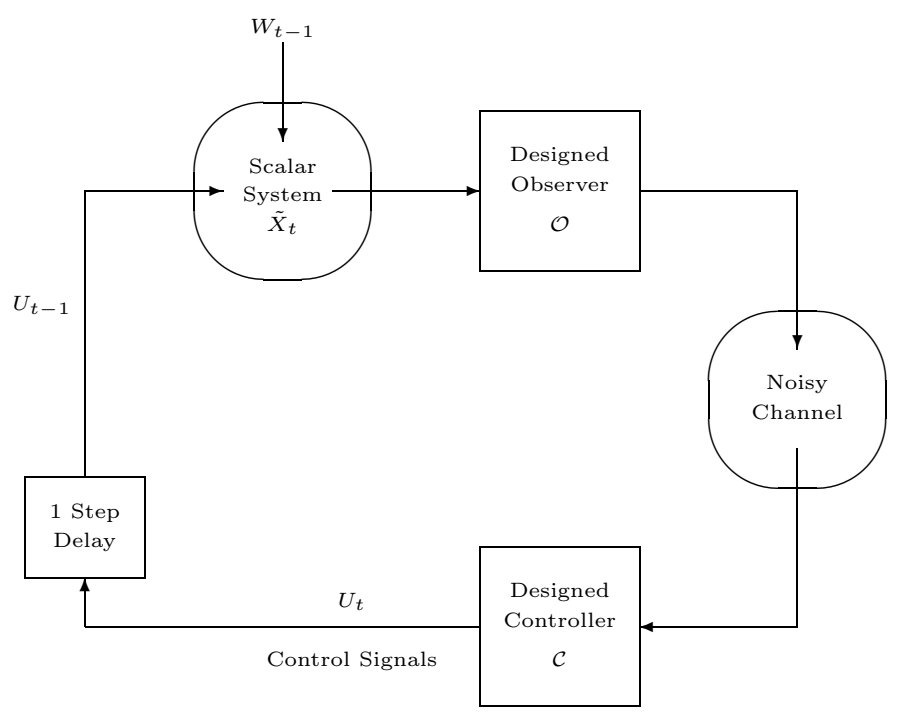

Figure 12: Control over a noisy communication channel without any explicit feedback path from controller to observer except through the plant.

potentially infinite bits of channel output information can be considered to be "below" the control bits. Since $L$ was arbitrary, we can choose it as large as we want.

\subsubsection{Removing noiseless access to the controls at the observer}

Theorem 6.4 still requires the observer to have perfect access to the control signals. It turns out that it is possible to eliminate that requirement and have the observer depend only on perfect access to the state $X_{t}$. After considering that case, we generalize it to having only boundedly noisy access to the state $X_{t}$ at the observer.

\subsubsection{Only perfect state observations $X_{t}$ available at observer}

The control information is needed at the observer to be able to work directly with the virtual process. We will give this information in two steps. First, we will make the plant dance in such a way that the observer can recover the channel output. With the most recent channel output in hand, the observer can combine it with past channel outputs to compute the applied control signal. The channel outputs will also be used for the feedback anytime code.

At time $t$ the observer can see the control signal only as it is corrupted by the process disturbance since $U_{t-1}+W_{t-1}=X_{t}-A X_{t-1}$. If we want noiseless information to flow from the controller to the observer, we can ask what is the instantaneous zero-error capacity of the effective channel through the plant. Here, the bounded support of $W$ and the unconstrained nature of $U$ become critical since they allow the instantaneous zero-error capacity of that channel to be infinite. Of course, there remains the problem of the dual-nature of the control signal - it is simultaneously being asked to stabilize the plant as well as to feedback information about the channel outputs. It turns out that the ability of the controller to move the plant provides enough feedback to the encoder in the case of finite channel output alphabets. 


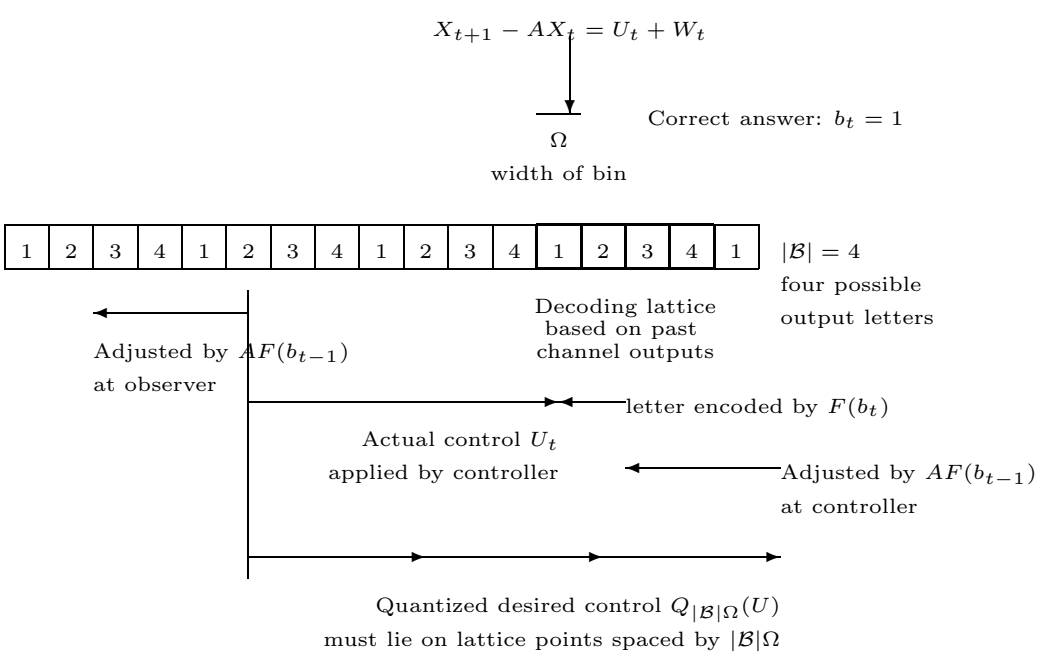

Figure 13: How to communicate the channel outputs through the plant. The controller restricts his main control signal to points on the lattice $|\mathcal{B}| \Omega$ and then precodes by $-A F\left(b_{t-1}\right)$ to eliminate the effect of the past writing. The final control signal applied is shifted slightly to encode which $b_{t}$ was received. The decoder uses the past $b_{t-1}$ to align its decoding lattice and then reads off $b_{t}$ by using $X_{t+1}-A X_{t}$.

Theorem 6.5 It is possible to control an unstable scalar process driven by a bounded disturbance over a noisy finite output alphabet channel so that the $\eta$-moment of $X_{t}$ stays finite for all time if the channel with feedback has $C_{a n y}(\alpha) \geq \log _{2} A$ for some $\alpha>\eta \log _{2} A$ if the observer is allowed to observe the state $X_{t}$ exactly.

Proof: The strategy is illustrated in figure 13. Let $|\mathcal{B}|$ be the size of the channel output alphabet. We use a modified control signal $U_{t}^{\prime \prime}=Q_{|\mathcal{B}| \Omega}\left(U_{t}\right)+F\left(b_{t}\right)-A F\left(b_{t-1}\right)$. Here $Q_{|\mathcal{B}| \Omega}$ quantizes its input to the nearest integer multiple of $|\mathcal{B}| \Omega$. $F$ maps the channel output into the range $\left(-\frac{|\mathcal{B}| \Omega}{2},+\frac{|\mathcal{B}| \Omega}{2}\right)$ as follows:

$$
F(b)=b \Omega-\frac{\Omega}{2}-\frac{|\mathcal{B}| \Omega}{2}
$$

Notice that $\min _{i \neq j}|F(j)-F(i)|=\Omega$ and so under a bounded disturbance no bigger than $\frac{\Omega}{2}$ in either direction we can always recover the channel output $B$ perfectly. By induction, the observer can remove $A F\left(b_{t-1}\right)$ and will then be able to extract both $Q_{|\mathcal{B}| \Omega}\left(U_{t}\right)$ and $F\left(b_{t}\right)$ noiselessly from $U_{t}^{\prime \prime}+W_{t}$. The net impact on the state will be finite — no more than $A|\mathcal{B}| \Omega$ in either direction — and so will not effect the qualitative tail probabilities.

At an abstract level, the controller is faced with the problem of causal "writing on dirty paper" [4] (or precoding) where the information it wishes to convey in one time step is the channel output and the dirty paper consists of the control signals it must apply to keep the system stable and to counteract the effect of the writing it did in previous time steps. The reason that we are able to solve the problem causally, without delay, and with zero-error is that there is no input magnitude constraint on us while the observation noise is bounded. 


\subsubsection{Observer has only noisy observations of the state}

Since the low-order bits of the state are already corrupted by the unknown disturbance $W$, it is clear that we do not need perfect access to the state. We can combine the result from corollary 5.1 with theorem 6.5. By combining $X_{\text {noisy }}(t)$ and $X_{\text {noisy }}(t+1)$ we can get $U_{t-1}+W_{t-1}+\left(N_{t}-A N_{t-1}\right)=$ $X_{\text {noisy }}(t)-A X_{\text {noisy }}(t+1)$. This is just as before except the observation of $U_{t-1}$ is corrupted by something with a bound of $\Omega+\Gamma(1+A)$ instead of just $\Omega$. All that matters is that it is bounded and so we can immediately write this corollary to theorem 6.5 .

Corollary 6.2 It is possible to control an unstable scalar plant driven by a bounded disturbance over a noisy finite output-alphabet channel so that the $\eta$-moment of $X_{t}$ stays finite for all time if the channel with feedback has $C_{\text {any }}(\alpha) \geq \log _{2} A$ for some $\alpha>\eta \log _{2} A$ if the observer is only allowed to observe the state $X_{t}$ corrupted by bounded noise.

Corollary 6.2 holds for any finite output alphabet channel regardless of its memory or other internal structure. This establishes that the necessity theorem 4.3 is effectively tight when all disturbances are bounded since the only gap between the two is the usual one between $>$ and $\geq$.

It might seem that this "dance" by the plant will be a disaster for performance. This is probably true, but it is important to realize that the goal here was only to address the question of stabilization and not the question of performance. We are not claiming that this particular dancing strategy is required for stabilization, just that it is sufficient.

\section{Continuous time systems}

\subsection{Notation specific to this section}

$X(t)$ will refer to a function of continuous time. $\dot{X}(t)$ refers to the usual first derivative of $X(t)$ with respect to time. In the context of a differential equation, it refers to the usual shorthand for the underlying integral equation. Data rates $R$ will be expressed in terms of nats rather than bits.

$\tau$ The sampling interval used to convert the continuous time system into a discrete time one.

$\tau_{c}$ The real time between channel uses in the continuous time model.

$\Omega^{\prime}$ The discrete-time translation of the continuous time bound on the disturbance magnitude.

\subsection{Overview}

So far, we have worked with a discrete-time model (1) for the dynamic system that we want to control over the communication link. This has allowed us to simplify the discussion by having a common clock that drives both the system and the uses of the noisy channel. In general, there will be a $\tau_{c}$ that represents the time between channel uses. This will allow us to translate everything into absolute time units.

$$
\dot{X}(t)=A X(t)+U(t)+W(t), \quad t \geq 0
$$

where we again assume a known bound on the disturbance $|W(t)| \leq \frac{\Omega}{2}$ and a known initial condition $X(0)=0$. We assume that the open loop system is unstable and hence: $A>0$.

We will use sampling to extend both our necessity and sufficiency results to the continuous time case. The basic result is that we will require an anytime capacity greater than $A$ nats per second. 


\subsection{Necessity}

For necessity, we are free to choose the disturbance signal $W(t)$ and as such, can restrict ourselves to piecewise constant signals ${ }^{33}$ that stay constant for time $\tau$. By sampling at the rate $\frac{1}{\tau}$ we find that

$$
X(\tau(i+1))=e^{A \tau} X(\tau i)+\left(\frac{e^{A \tau}-1}{A} W_{i}\right)+\int_{i \tau}^{(i+1) \tau} U(s) e^{A(\tau(i+1)-s)} d s
$$

Notice that (26) is just a discrete time system with $A^{\prime}=e^{A \tau}$ taking the role of $A$ in (1), and the disturbance is bounded by $\Omega^{\prime}=\frac{\Omega\left(e^{A \tau}-1\right)}{A}$. All that remains is to reinterpret our earlier theorem.

Set $\tau=\tau_{c}$ to match up the sampling time for the continuous time system to the channel time and we immediately get that the appropriate anytime capacity must exceed $\log _{2} A^{\prime}=\tau_{c} A \log _{2}$ e bits per channel use. If we convert units to nats per second ${ }^{34}$, we get the intuitively appealing result that the anytime capacity must be greater that $A$ nats $/$ sec $^{35}$ Similarly, to hold the $\eta$-th moment constant, we need the probability of error to drop with delay as $K 2^{-\left(\eta A \log _{2} e\right) d \tau_{c}}$ where $d$ is in units of channel uses and thus $d \tau_{c}$ has units of seconds. Thus we get the following pair of theorems:

Theorem 7.1 For a given noisy channel and $\eta>0$, if there exists an observer $\mathcal{O}$ and controller $\mathcal{C}$ for the unstable scalar continuous time system that achieves $E\left[|X(t)|^{\eta}\right]<K$ for all $t$ and bounded driving noise signals $|W(t)| \leq \frac{\Omega}{2}$, then $C_{a n y}\left(\eta A \log _{2} e\right) \geq A$ nats per second for the noisy channel considered with the encoder having access to noiseless feedback.

Theorem 7.2 For a given noisy channel and monotonic decreasing function $f(m)$, if there exists an observer $\mathcal{O}$ and controller $\mathcal{C}$ for the unstable continuous-time scalar system that achieves $\mathcal{P}(|X(t)|>$ $m)<f(m)$ for all $t$ and bounded driving noise signals $|W(t)| \leq \frac{\Omega}{2}$, then $C_{g \text {-any }}(g) \geq A$ nats per second for the noisy channel considered with the encoder having access to noiseless feedback and $g(d)$ having the form $g(d)=f\left(K e^{A d}\right)$ for some constant $K$.

\subsection{Sufficiency}

For sufficiency, we are free to sample the signal as we want at the observer and apply piecewise constant control signals. Sampling at $\tau$ gives rise to (26) only with the roles of $W$ and $U$ reversed. We can see that $W_{i}=\int_{i \tau}^{(i+1) \tau} W(s) e^{A(\tau(i+1)-s)} d s$ is still bounded by substituting in the upper and lower bounds and then noticing that $\left|W_{i}\right| \leq \frac{\Omega\left(e^{A \tau}-1\right)}{2 A}$.

As such, the same argument as necessity above holds and we can just translate the sufficiency theorems 5.1, 5.2, 6.1, 6.4, and 6.5 into continuous time. In each, the feedback anytime capacity must be greater than $A$ nats per second. Since the necessary and sufficient conditions are right next to each other, it is clear that we can do no better or worse in terms of stabilization by choosing a different sampling time $\tau$. Of course, this need not be optimal in terms of performance beyond stabilization.

\footnotetext{
${ }^{33}$ zero order hold

${ }^{34}$ Assuming that $\dot{X}$ was in per second units.

${ }^{35}$ This truly justifies nats as the "natural" unit of information!
} 


\section{Vector-valued systems (Part II)}

\subsection{Notation specific to this section}

Vector valued variables will usually be marked as $\vec{X}$. The $i$-th component will be $X_{i} . X_{i}(t)$ refers to the $i$-th component of $\vec{X}_{t}$.

$A$ The unstable system dynamics - matrix valued.

$B_{u}, B_{w}$ The input transforming matrices corresponding to the control and disturbance inputs correspondingly

$C_{y}$ The observation matrix - tells what linear combinations of the underlying state $\vec{X}$ are actually available at the observer $\mathcal{O}$.

$n$ The dimensionality of $\vec{X}$. Because of controllability and observability issues, it is also used to consider time in blocks.

$m_{u}, m_{w}, m_{y}$ The dimensionalities of the $\vec{U}, \vec{W}, \vec{Y}$.

$\vec{U}_{t}$ The vector control applied to the vector system at the end of time $t$.

$\vec{U}_{t}^{\prime}$ The transformed vector control. It acts on each component separately.

$\vec{W}_{t}$ The bounded vector disturbance entering the vector system at the end of time $t$.

$\vec{W}_{t}^{\prime}$ The transformed vector disturbance. It acts on each component separately.

$\vec{X}_{t}$ The vector plant state at time $t$.

$\vec{X}_{t}^{\prime}$ The transformed vector state so that each component has independent dynamics.

$R_{i}$ For multi-stream encoding, the rate of the $i$-th parallel bitstream.

$\mathcal{R}_{\text {any }}(\vec{\alpha})$ The rate region supported by a channel when the individual bitstreams must satisfy the anytime reliabilities $\alpha_{i}$.

$\vec{\alpha}$ A vector of anytime reliabilities $\alpha_{i}$ for different bitstreams being carried over a single noisy channel.

$\Delta_{i}$ The size of the box that the observer attempts to keep the $i$-th component of the state in.

$\lambda_{i}, \vec{\lambda}$ The $i$-th unstable eigenvalue and the vector of all unstable eigenvalues.

$\Lambda$ A diagonal matrix consisting only of the eigenvalues of $A$.

$\Lambda^{\prime}$ A diagonal matrix consisting only of the magnitudes of the eigenvalues of $A$.

$\Omega_{i}$ The effective bound on the disturbance that is used when trying to encode the $i$-th component of the transformed state corresponding to a non-diagonal Jordan block.

$\Omega^{\prime}$ The inscribed bound for the $\vec{W}^{\prime}$

$\bar{\Omega}$ The circumscribed bound for the $\vec{W}^{\prime}$ 


\subsection{Overview}

With the scalar case done, we next discuss the case of vector-valued systems. As the issues of going to continuous-time are identical to those encountered in the scalar case, we will focus on the discrete-time case here. In cases with vector-valued state, three fundamental issues arise from possible mismatches in dimensionality.

- The observation vector available to the encoder might be of lower dimensionality than the state. This will require dealing with the concept of observability.

- The control vector might be of lower dimensionality than the state. This will require dealing with the concept of controllability.

These first two can be dealt with the traditional linear systems machinery. In our context, there is another one which is not immediately apparent:

- The bounded disturbance vector might be of lower dimensionality than the state. This will require a second demand of controllability while proving necessity.

Our model follows (1) except that everything is vector-valued now:

$$
\vec{X}_{t+1}=A \vec{X}_{t}+B_{u} \vec{U}_{t}+B_{w} \vec{W}_{t}, \quad t \geq 0
$$

where $\left\{\vec{X}_{t}\right\}$ is an $\mathbb{R}^{n}$-valued state process $\left\{\vec{U}_{t}\right\}$ is an $\mathbb{R}^{m_{u}}$-valued control process and $\left\{\vec{W}_{t}\right\}$ is a bounded noise/disturbance process taking values in $\mathbb{R}^{m_{w}}$ s.t. $\left\|\vec{W}_{t}\right\| \leq \frac{\Omega}{2}$ where we can use any finitedimensional norm that we find convenient. For convenience, we also assume a known initial condition $\vec{X}_{0}=\overrightarrow{0}$.

In addition, we will allow for the input to the encoder to be a linear function of the state, rather than the state itself.

$$
\vec{Y}_{t}=C_{y} \vec{X}_{t}
$$

where $\vec{Y}_{t}$ is an $m_{y}$ dimensional vector. The matrices $A, B_{u}, B_{w}, C_{y}$ above are all of the appropriate dimensionality so that the equations (27) and (28) make sense. We know that even without any communication constraint, for us to be able to stabilize the system, we generally require $\left(A, B_{u}\right)$ to be a controllable pair and $\left(A, C_{y}\right)$ to be an observable pair. ${ }^{36}$

In addition to the standard requirement that $\left(A, B_{u}\right)$ be controllable ${ }^{37}$ and $\left(A, C_{y}\right)$ be observable ${ }^{38}$, we will make an additional requirement that $\left(A, B_{w}\right)$ is controllable when determining necessary conditions for stabilization. This is a reasonable assumption as it corresponds to requiring that the disturbance can repeatedly excite all the unstable modes of the system. Otherwise, in the context of the assumption of known initial conditions asserting that $\vec{X}_{0}=\overrightarrow{0}$, those unstable modes may never get excited.

\footnotetext{
${ }^{36}$ We will not worry about the slight distinction between controllable and stabilizable here. The modes of the linear system that are already stable are not going to be causing us trouble. If any such uncontrollable stable modes exist, let them.

${ }^{37} \mathrm{~A}$ pair of matrices $(A, B)$ are controllable if the matrix $\left[B, A B, A^{2} B, \ldots, A^{n-1} B\right]$ is of full rank. This condition assures us that by appropriate choice of inputs, we can control the behavior of all the modes of the linear dynamical system.

${ }^{38} \mathrm{~A}$ pair of matrices $(A, C)$ are observable if the matrix $\left[C, C A, C A^{2}, \ldots, C A^{n-1}\right]^{T}$ is of full rank. This condition assures us that by combining enough raw observations, we can see the behavior of all the modes of the linear dynamical system.
} 
In section 8.3, we study the case of $A$ matrices that are real and diagonal. This is essentially multiple copies of the scalar case and it lets us see what the requirements are going to be in the vector case with a minimum of technical difficulties. In section 8.4, we move to the generic case of vector systems that have diagonalizable $A$ matrices. Here, the issues of controllability and observability become important, but can be addressed by treating time in the appropriate blocks. There is also a more subtle issue regarding intrinsic delays in control systems that is dealt with in section 8.5. Finally, in section 8.6, the nongeneric case of nondiagonalizable matrices is addressed.

\subsection{The real diagonal case}

We first consider the simplest possible case of a vector problem: where the real $A$ matrix is diagonal with eigenvalues $\lambda_{i}$ for $i=1 \ldots n$. The controllability and observability assumptions translate into $B_{u}, B_{w}, C_{y}$ all being full-rank. As such, we can change variables slightly by substituting $\vec{U}_{t}=B_{u}^{-1} \vec{U}_{t}^{\prime}$, $\vec{W}_{t}=B_{w}^{-1} \vec{W}_{t}^{\prime}$, and using $X_{t}=C_{y}^{-1} Y_{t}$ directly as our observation. In this case the problem decomposes into $L$ parallel scalar problems:

$$
X_{i}(t+1)=\lambda_{i} X_{i}(t)+U_{i}^{\prime}(t)+W_{i}^{\prime}(t)
$$

for $i=1 \ldots N$ with the only connection between the different parallel problems $i$ being the common restriction $\left\|B_{w}^{-1} \vec{W}_{t}^{\prime}\right\| \leq \frac{\Omega}{2}$. However, since all finite-dimensional norms are equivalent, we are free to translate that restriction into a pair of restrictions:

- For the necessity part, where we need to generate $\vec{W}_{t}$ signals to carry data across the channel, we can use an inscribed hyper-cube within the $B_{w}^{-1}$ mapped $\frac{\Omega}{2}$ sized ball to allow ourselves to pick appropriate disturbances to the parallel control systems without worrying about what that means for other ones. This can be done since $\exists \Omega^{\prime}$ s.t. $\left\|\vec{W}^{\prime}\right\|_{\infty} \leq \frac{\Omega^{\prime}}{2}$ implies $\left\|B_{w}^{-1} \vec{W}_{t}^{\prime}\right\| \leq \frac{\Omega}{2}$. So, for the necessity theorems, the scalar proofs above apply with $\Omega^{\prime}$ playing the part of the bound.

- For the sufficiency proofs, we require a bounded range that the disturbance can take on system $i$. Again, we can use the equivalency of the $\infty$-norm to all other finite-dimensional norms and inscribe the $B_{w}^{-1}$ mapped $\frac{\Omega}{2}$ sized ball from the original norm inside a hyper-cube $\bar{\Omega}$ on each side.

This same equivalency between norms tells us that if we have a finite $\eta$-moment of the $\infty$-norm of $\vec{X}$, then we also have a finite $\eta$-moment of any other norm and vice versa. ${ }^{39}$ Furthermore, we have following lemma showing that if a finite collection of random variables all have finite $\eta$-moments, then they have a finite $\eta$-moment when collected together into a vector and vice versa.

Lemma 8.1 Let $\left|X_{i}\right|$ be positive random variables. There exists a $K$ such that $E\left[\left|X_{i}\right|^{\eta}\right] \leq K$ for each $i$ if and only if there exists a $K^{\prime}$ such that $E\left[\|\vec{X}\|^{\eta}\right] \leq K^{\prime}$.

Furthermore, if $E\left[\|\vec{X}\|^{\eta}\right] \leq K$, then for any matrix $L$ there exists $K^{\prime \prime}$ so that $E\left[\|L \vec{X}\|^{\eta}\right] \leq K^{\prime \prime}$.

\footnotetext{
${ }^{39}$ Whatever constant factor that bounds the norm from the $\infty$-norm will just be raised to the $\eta$-th power. This will change the finite expectation by at most that factor and hence finite expectations will remain finite.
} 
Proof: First we assume that $E\left[\|\vec{X}\|^{\eta}\right] \leq K^{\prime}$. Then we know by the equivalencies of finite-dimensional norms that:

$$
\begin{aligned}
K^{\prime} & \geq E\left[\|\vec{X}\|^{\eta}\right] \\
& \geq \kappa^{\eta} E\left[\|\vec{X}\|_{\infty}^{\eta}\right] \\
& =\kappa^{\eta} E\left[\left(\max _{i}\left|X_{i}\right|\right)^{\eta}\right] \\
& \geq \kappa^{\eta} E\left[\left|X_{i}\right|^{\eta}\right]
\end{aligned}
$$

So we have $\left.E\left[\left|X_{i}\right|\right)^{\eta}\right] \leq \frac{K^{\prime}}{\kappa^{\eta}}$. To see the other direction, first assume that $E\left[\left|X_{i}\right|^{\eta}\right] \leq K$.

$$
\begin{aligned}
E\left[\|\vec{X}\|^{\eta}\right] & \leq \kappa^{\prime \eta} E\left[\|\vec{X}\|_{\infty}^{\eta}\right] \\
& =E\left[\left(\max _{i}\left|X_{i}\right|\right)^{\eta}\right] \\
& =\int_{0}^{\infty} \mathcal{P}\left(\left(\max _{i}\left|X_{i}\right|\right)^{\eta} \geq t\right) d t \\
& \left.\leq \int_{0}^{\infty} \sum_{i} \mathcal{P}\left(\left|X_{i}\right|\right)^{\eta} \geq t\right) d t \\
& \left.=\sum_{i} \int_{0}^{\infty} \mathcal{P}\left(\left|X_{i}\right|\right)^{\eta} \geq t\right) d t \\
& =\sum_{i} E\left[\left|X_{i}\right|^{\eta}\right] \\
& \leq n K
\end{aligned}
$$

For the second part, we use the $\infty$-norm again and given the previous part, we just need to consider a single term $\sum_{i=1}^{n} l_{i} X_{i}$ and show that its $\eta$-moment is bounded.

$$
\begin{aligned}
E\left[\left|\sum_{i=1}^{n} l_{i} X_{i}\right|^{\eta}\right] & \leq E\left[\left(\sum_{i=1}^{n}\left|l_{i} X_{i}\right|\right)^{\eta}\right] \\
& \leq E\left[\left(\sum_{i=1}^{n}\left(\max _{i}\left|l_{i}\right|\right)\left(\max _{i}\left|X_{i}\right|\right)\right)^{\eta}\right] \\
& \leq\left(n\|\vec{l}\|_{\infty}\right)^{\eta} E\left[\|\vec{X}\|_{\infty}^{\eta}\right] \\
& \leq\left(n\|\vec{l}\|_{\infty}\right)^{\eta} K^{\prime \prime}
\end{aligned}
$$

which proves the result.

Using lemma 8.1 and the discussion above, we can immediately translate theorem 4.3 on a component-wise basis. Before we state the appropriate corollary, we introduce the notion of an anytime rate region that generalizes the notion of a single anytime capacity to a rate region corresponding to a vector of anytime-reliabilities specifying how fast the different bitstreams have their probabilities of error tending to zero with delay.

Definition 8.1 The anytime rate region $\mathcal{R}_{a n y}(\vec{\alpha})$ of a channel is the set of rate-tuples which the channel can support so that each has a probability of error that decays at least exponentially with delay at a rate $\alpha_{i}$. There has to exist a uniform constant $K$ such that for each $i$, all $d$ and all times $t$ we have

$$
\mathcal{P}\left(\hat{M}_{i, 1}^{t-d}(t) \neq M_{i, 1}^{t-d}(t)\right)<K 2^{-\alpha_{i} d}
$$


where the $M_{i, j}$ corresponds to the $j$-th message sent in the $i$-th bitstream.

The idea of the anytime rate region here is to transport parallel bitstreams across the noisy channel, giving each one a potentially different level of reliability. This is related to the idea of differentiated service in networks and was explored in the control context in [20] where it was shown by a diagonal example that certain reliability tradeoffs are only achievable if the anytime encoder actively discriminates among the bitstreams.

Corollary 8.1 For a given noisy channel, diagonal $A$ with diagonal elements $\lambda_{i}>1$, bound $\Omega$, and $\eta>0$, if there exists an observer $\mathcal{O}$ and controller $\mathcal{C}$ for the unstable vector system that achieves $E\left[\left\|\vec{X}_{t}\right\|^{\eta}\right]<K$ for all sequences of bounded driving noise $\left\|\vec{W}_{t}\right\| \leq \frac{\Omega}{2}$, then for every $\vec{\epsilon}>0$ we know that $\left(\log _{2} \vec{\lambda}-\vec{\epsilon}\right) \in \mathcal{R}_{\text {any }}\left(\eta \log _{2} \vec{\lambda}\right)$ for the noisy channel considered with the encoder having access to noiseless feedback.

The $\log _{2} \vec{\lambda}$ is shorthand for the vector consisting of the base two logarithms of all the components of $\vec{\lambda}$. Similarly, we can carry over the sufficiency results for the real diagonal case to get the basic vector corollary to theorem 5.2 :

Corollary 8.2 It is possible to control an unstable vector process with diagonal controllable dynamics and unstable eigenvalues $\vec{\lambda}$ driven by a bounded disturbance over a noisy channel so that the $\eta$ moment of $\left\|\vec{X}_{t}\right\|$ stays finite for all time if the channel with noiseless feedback has $\left(\log _{2}(\vec{\lambda})+\vec{\epsilon}\right) \in$ $\mathcal{R}_{\text {any }}\left(\eta \log _{2} \vec{\lambda}+\vec{\epsilon}\right)$ for some $\vec{\epsilon}>0$ and the observer is allowed to observe the noisy channel outputs.

The same carrying over can be done for theorems $6.1,6.4$, and 6.5 as well. The situation with generalizing corollary 6.1 is slightly more interesting. Since all we want is to have some $\eta$ for which the system is stable, we do not need to differentiate the service among the dimensions. We can operate at a rate larger than the sum of the logs of the unstable eigenvalues and then translate the resulting anytime reliability into a particular bound $\eta$.

Corollary 8.3 For diagonal controllable dynamics, if the observer is allowed boundedly noisy access to the vector plant state, and the noisy channel is a DMC with Shannon capacity $C>\sum_{i} \log _{2}\left|\lambda_{i}\right|$, then there exists an observer/controller pair that stabilizes the system in closed loop so that the $\eta$ moment of $\vec{X}_{t}$ stays finite for all time for some $\eta>0$ as long as the observer/controller are allowed to share common randomness.

\subsection{Diagonal Jordan forms}

We next show how we can extend the results for real diagonal $A$ matrices to the case of linear systems that have real $A$ matrices that have diagonal Jordan forms - i.e. have a full complement of eigenvectors. This represents a generic $A$ matrix since the matrices that do not have a full complement of eigenvectors form a measure-zero set. The goal here is to show that the magnitudes of the unstable eigenvalues are all that matter. The two main tools will be to look at time in blocks and to change coordinates to diagonalize the system.

Theorem 8.2 Assume that for a given noisy channel, system dynamics described by (27) with diagonalizable $A$ and eigenvalues $\lambda_{i}$, and $\eta>0$, that there exists an observer $\mathcal{O}$ and controller $\mathcal{C}$ for the unstable vector system that achieves $E\left[\left\|\vec{X}_{t}\right\|^{\eta}\right]<K$ for all sequences of bounded driving noise $\left\|\vec{W}_{t}\right\| \leq \frac{\Omega}{2}$. Furthermore, assume that the pair $\left(A, B_{w}\right)$ is controllable. 
Let $\left|\lambda_{i}\right|>1$ for $i=1 \ldots l$, and let $\vec{\lambda}$ be the l-dimensional vector consisting of only the exponentially unstable eigenvalues ${ }^{40}$ of $A$. Then for every $\vec{\epsilon}>0$ we know that $\left(\log _{2} \vec{\lambda}_{\|}-\vec{\epsilon}\right) \in \mathcal{R}_{\text {any }}\left(\eta \log _{2} \vec{\lambda}_{\|}\right)$for the noisy channel considered with the encoder having access to noiseless feedback. $\vec{\lambda}_{\|}$is the vector whose components are the magnitudes of the unstable eigenvalues.

Proof: We want to turn the problem into one for which corollary 8.1, the case of diagonal real matrices, applies.

Recall that at the encoder side, the disturbance input is used to embed our data bits into the uncontrolled state. Since $\left(A, B_{w}\right)$ is a controllable pair, we know that within at most $n$ time steps, it is possible to take the system starting at $\vec{X}_{t}=\overrightarrow{0}$ and drive it to any $\vec{X}_{t+n+1}=\vec{v}$ through the choice of disturbance inputs $\vec{U}_{t}^{t+n+1}$. Since the system is linear, given a bound on $\|\vec{U}\|_{\infty}$, we can determine an $\Omega^{\prime}$ so that as long as $\|\vec{v}\|_{\infty}<\frac{\Omega^{\prime}}{2}$ we can choose an acceptable sequence of $n$ disturbance vectors to reach it.

As such, we can use linearity and rewrite the system dynamics (27) using blocks of time of size $n$ as:

$$
\vec{X}_{n k}=A^{n} \vec{X}_{n(k-1)}+\vec{U}_{k}^{\prime}+\vec{W}_{k}^{\prime}, \quad t \geq 0
$$

where $\vec{U}_{k}^{\prime}$ just combines the net effect of the controls over the past $n$ time-steps and $\vec{W}_{k}^{\prime}$ is now an arbitrary $n$-vector disturbance with $\left\|\vec{W}_{k}^{\prime}\right\|_{\infty} \leq \frac{\Omega^{\prime}}{2}$.

Since $A$ is diagonalizable, we know that there exists a nonsingular matrix $V$ so that $V A V^{-1}=\Lambda$, a diagonal matrix with the first $l$ diagonal elements corresponding to the exponentially unstable eigenvalues. Thus $A^{n}=V^{-1} \Lambda^{n} V$. To avoid any complications arising from the complex eigenvalues, we can use a standard trick[34] and use a rotating coordinate frame where $\vec{X}_{k n}^{\prime}=\left(U_{k n} V\right) \vec{X}_{k n}$ so that $\left(U_{k n} V\right)$ is a real time-varying matrix transforming (30) into:

$$
\vec{X}_{n k}^{\prime}=\Lambda^{\prime} \vec{X}_{n(k-1)}^{\prime}+\vec{U}_{k}^{\prime \prime}+\vec{W}_{k}^{\prime \prime}, \quad t \geq 0
$$

where $\Lambda^{\prime}$ is a real diagonal matrix with the $i$ th entry being $\left|\lambda_{i}\right|^{n}$. The $\vec{U}^{\prime \prime}$ and $\vec{W}^{\prime \prime}$ are essentially the same as they were in (30) except possibly with a smaller constant bound $\Omega^{\prime \prime}>0$ due to the rotating change of basis.

Now the second part of lemma 8.1 can deal with the change of coordinates and we can apply corollary 8.1 to the unstable subspace of (31). We observe that $\log _{2}\left|\lambda_{i}\right|^{n}=n \log _{2}\left|\lambda_{i}\right|$ and by expressing both the delay in unit time rather than in steps of $n$ and the bitrates per unit time rather than in steps of $n$, we have the desired result.

The same ideas can be used to extend the sufficiency result from corollary 8.2 to give us:

Theorem 8.3 Assume that we have a noisy channel with access to noiseless feedback with an anytime rate region for some $\vec{\epsilon}>0$ that has $\left(\log _{2}\left(\vec{\lambda}_{\|}\right)+\vec{\epsilon}\right) \in \mathcal{R}_{\text {any }}\left(\eta \log _{2} \vec{\lambda}_{\|}+\vec{\epsilon}\right)$ where $\vec{\lambda}_{\|}$consists of the component-wise magnitudes of the $\vec{\lambda}$.

Then we can stabilize the linear system with dynamics described by (27) with diagonalizable A, unstable eigenvalues $\vec{\lambda}$, controllable $\left(A, B_{u}\right)$, observable $\left(A, C_{y}\right)$, bound $\Omega$, by constructing an observer $\mathcal{O}$ and controller $\mathcal{C}$ for the unstable vector system that achieves $E\left[\left\|\vec{X}_{t}\right\|^{\eta}\right]<K$ for all sequences of bounded driving noise $\left\|\vec{W}_{t}\right\| \leq \frac{\Omega}{2}$ if the observer has access to the noisy channel outputs.

\footnotetext{
${ }^{40}$ eigenvalues with multiplicity should appear multiple times in $\vec{\lambda}$
} 
Proof: First, we consider the case where we can observe $\vec{X}_{t}$ directly at the encoder. Using the same ideas as the proof of theorem 8.2, we can setup a rotating coordinate frame in which the plant behaves as though it were real and diagonal. However, there is still the issue of choosing control signals. Once again, we can use controllability, this time of $\left(A, B_{u}\right)$ and treat time in blocks of $n$ at a time. Since there is no constraint on the $U_{t}$, we know that the controller can act in a way to completely subtract the effect of $\hat{X}_{t-n}$ from $X_{t}$. So we have reduced the problem to that addressed in corollary 8.2.

Now, consider the case where $\vec{X}_{t}$ is not observed directly by the encoder and only $\vec{Y}_{t}$ is available. We use the observability of $\left(A, C_{y}\right)$. This tells us that with the $n$ observations ${ }^{41}\left(Y_{n k}^{n(k+1)-1}, U_{n k}^{n(k+1)-1}\right)$ we can determine $\vec{X}_{n k}$, except for the linear effect of the unknown disturbances $\vec{W}_{n k}^{n(k+1)-1}$. Since the disturbance is bounded, so is the effect of the linear combination of observations used to extract $\vec{X}_{n k}$. Consider this a bounded observation noise and use corollary 5.1 as generalized straightforwardly to the diagonal case.

\subsection{Intrinsic delay and relaxing feedback to observers}

By extending the strategy of the previous sections straightforwardly to blocks of $2 n$, we can enforce no control inputs for the first half, and use that time to do observations on $\vec{X}_{t}$ through the $\vec{Y}_{t}$ at the observer. During the second half of the block, we can apply controls based on the best information received so far. This exploits the controllability. By this strategy, the problem of diagonalizable Jordan blocks reduces entirely to $n$ parallel scalar problems, each one observed with noisy observations of the exact state. As such, all of the scalar results on sufficiency will seem to generalize to the vector case, including those that have no explicit access to the channel feedback at the observer.

However, the reduction to $n$ scalar problems comes along with a delay of $2 n$ in communication back from the controller to the observer through the plant. Since the channel feedback will also be delayed by $2 n$, the sense of anytime reliability required for sufficiency is much stronger than what is required in theorem 8.3. In this section, we give an example that shows that some added delay can be unavoidable and then define what the intrinsic delay is for a linear system. We then use this concept to give more refined necessity and sufficiency theorems that are tight and have additional delays in feedback.

The following example shows that this is not purely an artifact of the proof technique and that some delay can in fact be intrinsic to the vector case.

Example 8.1 Consider the single input single output (SISO) system described by:

$$
\begin{gathered}
A=\left[\begin{array}{lll}
2 & 1 & 0 \\
0 & 3 & 1 \\
0 & 0 & 4
\end{array}\right] \\
B_{u}=\left[\begin{array}{l}
0 \\
0 \\
1
\end{array}\right] \\
C_{y}=[1,0,0]
\end{gathered}
$$

It controllable since $\left[B, A B, A^{2} B\right]$ is full rank and observable since $\left(C, C A, C A^{2}\right)$ also span three dimensions. Since the eigenvalues 2,3,4 are all distinct, it is certainly diagonalizable as well.

\footnotetext{
${ }^{41}$ From the noiseless feedback of channel outputs, we can reconstruct the control signals as well.
} 
For such a system, initialized to 0 , the impulse response is $0,0,1,9, \ldots$. The input is not immediately visible at the output and takes 3 total time steps to show up at all. There is no possible way to communicate through the plant with only unit delay.

Example 8.1 motivates the following definition:

Definition 8.4 The intrinsic delay $\Theta\left(A, B_{u}, C_{y}\right)$ of a linear system is the amount of time it takes the input to become visible at the output. It is the minimum integer ${ }^{42} i \geq 0$ for which $C_{y} A^{i} B_{u} \neq 0$.

For SISO systems, this is just the position of the first nonzero entry in the impulse response.

\subsubsection{Refined necessity theorem}

This definition of intrinsic delay allows us to state a more refined version of theorem 8.2:

Theorem 8.5 Assume that for a given noisy channel, system dynamics described by (27) with diagonalizable $A$ and eigenvalues $\lambda_{i}$, and $\eta>0$, that there exists an observer $\mathcal{O}$ and controller $\mathcal{C}$ for the unstable vector system that achieves $E\left[\left\|\vec{X}_{t}\right\|^{\eta}\right]<K$ for all sequences of bounded driving noise $\left\|\vec{W}_{t}\right\| \leq \frac{\Omega}{2}$. Furthermore, assume that the pair $\left(A, B_{w}\right)$ is controllable.

Then for every $\vec{\epsilon}>0$ we know that $\left(\log _{2} \vec{\lambda}_{\|}-\vec{\epsilon}\right) \in \mathcal{R}_{\text {any }}\left(\eta \log _{2} \vec{\lambda}_{\|}\right)$for the noisy channel considered with the encoder having access to noiseless feedback delayed by $1+\Theta\left(A, B_{u}, C_{y}\right)$.

Proof: In order to generate the next simulated $\vec{Y}_{t+1}$ to feed to the observer $\mathcal{O}$, we only need the exact $\vec{U}$ values upto time $t-\Theta\left(A, B_{u}, C_{y}\right)$ since controls after that point have not become visible yet. Thus, we can live with noiseless feedback that has $1+\Theta\left(A, B_{u}, C_{y}\right)$ delay rather than the unit delay assumed earlier.

\subsubsection{Refined sufficiency theorem}

To close the gap entirely, we need to show how to communicate the exact channel output back to observer through the plant using only $\Theta$ time steps. This will give:

Theorem 8.6 Assume that we have a noisy finite-output-alphabet channel such that with access to noiseless feedback delayed by $1+\theta$ time units, it has an anytime rate region for some $\vec{\epsilon}>0$ that has $\left(\log _{2}\left(\overrightarrow{\lambda_{\|}}\right)+\vec{\epsilon}\right) \in \mathcal{R}_{\text {any }}\left(\eta \log _{2} \vec{\lambda}_{\|}+\vec{\epsilon}\right)$ where $\vec{\lambda}_{\|}$consists of the component-wise magnitudes of the $\vec{\lambda}$.

Then we can stabilize the linear system with dynamics described by (27) with diagonalizable $A$, unstable eigenvalues $\vec{\lambda}$, controllable $\left(A, B_{u}\right)$, observable $\left(A, C_{y}\right)$, intrinsic delay $\Theta\left(A, B_{u}, C_{y}\right)=\theta$, by constructing an observer $\mathcal{O}$ and controller $\mathcal{C}$ for the unstable vector system that achieves $E\left[\left\|\vec{X}_{t}\right\|^{\eta}\right]<$ $K$ for all sequences of bounded driving noise $\left\|\vec{W}_{t}\right\| \leq \frac{\Omega}{2}$ if the observer has access to the observations $Y_{t}$ corrupted by bounded noise.

Proof: We will show how to patch together the proofs of theorem 8.3, theorem 6.5, and its corollary 6.2. The key idea is illustrated in figure 14 .

In the proof of theorem 8.3, we used the observer's knowledge of the channel outputs for two purposes. First, it was used in the operation of the anytime code. Second, it was used to compute

\footnotetext{
${ }^{42}$ Technically speaking, this should be a theorem rather than part of the definition, but since it is an obvious consequence of linearity, we just put it in the definition itself for convenience.
} 


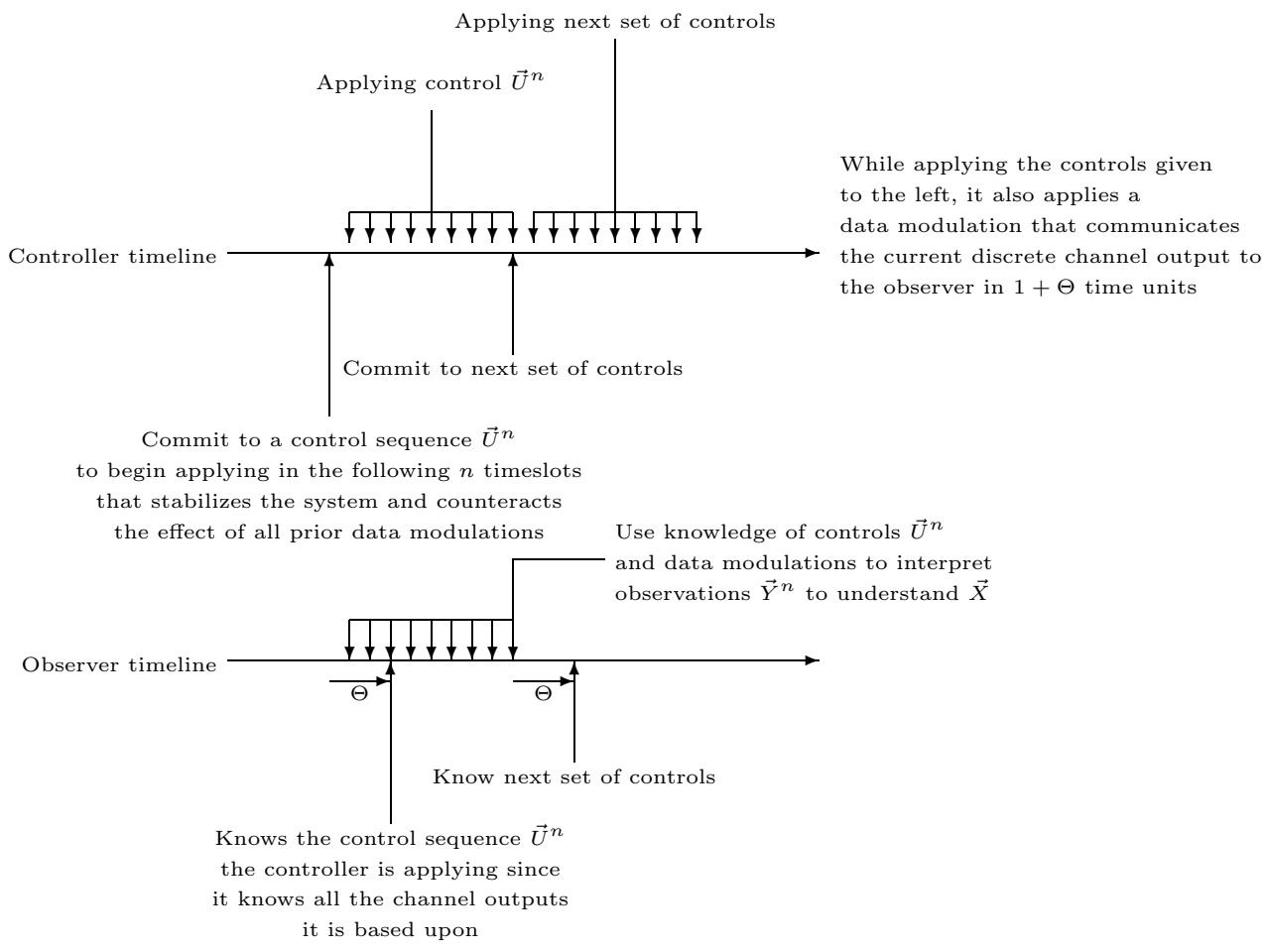

Figure 14: When viewing time in blocks of $n$, we require the controller to commit to its primary controls 1 time step before actually putting them into effect. This way, by the time the observer can first see the effect of the control strategy, it already knows exactly what it is going to be since it knows all the channel outputs that it was based upon. 
what the control signal was so that the observer could take it into account while observing $\vec{X}_{n k}$. Here, we need the channel outputs at time $1+\theta$ for running the anytime code. Since $\theta \leq n$, by the time the observer has seen enough $\vec{Y}$ to observe $\vec{X}_{n k}$, it will also have access to the channel outputs that were used to compute the controls applied during that period. So, it can still subtract the effect of the controls while interpreting its observations.

To actually communicate the channel outputs from the controller to the observer, we follow the strategy from the proof of theorem 6.5 with the following modifications:

- The observer will use some component $Y_{i}(t+1)$ of $\vec{Y}_{t+1}$ that is potentially impacted by $\vec{U}_{t-\theta}$ to read the value of $B_{t-\theta}$. This will take the place of $X_{t+1}$ when it comes time to think of the lattice coding.

- By that time (by induction), the observer knows exactly what the values were for all the $B_{1}^{t-\theta-1}$ and so it already knows all the control signals except that part of $\vec{U}_{t-\theta}$ that will be used to communicate $B_{t-\theta}$. It can therefore remove their entire impact from the output $Y_{i}(t+1)$.

- The $\vec{Y}_{1}^{t-1}$ and known controls so far give an estimate of $\vec{X}_{t-n}$ that differs from the true $\vec{X}_{t-n}$ by no more than some bound $\Gamma^{\prime}$ in any component. Through the linear operations of the observer, this translates into some constant $\Gamma^{\prime \prime}$ of prior uncertainty on $Y_{i}(t+1)$.

- $\Gamma^{\prime \prime}$ can thus play the role of $\Omega$ in the lattice encoding figure 13 . The encoder can thus encode the value of $B_{t-\theta}$ by modulating this data on top of $\vec{U}_{t-\theta}$ so that it causes a shift of an integer multiple of $\Gamma^{\prime \prime}$ guaranteed to be visible in $Y_{i}(t+1)$.

- At the time the controller commits to a primary control sequence $\vec{U}_{k n+1}^{k n+n}$ for the next $n$ control signals, it can also clean up for all the past data modulations that it has done to communicate past channel outputs. This way, the closed-loop system will stay stable since the deviations in the controls used to communicate will not have an impact that is allowed to propagate unstably through the system.

Everything else is as before.

\subsubsection{Comments}

Theorems 8.5 and 8.6 show that in the vector case, there can be something fundamentally harder about stabilizing an unstable process when explicit noiseless unit-delay feedback of the channel is not available. If the input-output behavior of the system has an intrinsic delay associated with it, then we require that the noisy channel support enough anytime-reliability at the target rates even with feedback delayed by that intrinsic amount. Knowing the unstable eigenvalues and target moment $\eta$ is not enough to evaluate a noisy channel.

\subsection{Non-diagonal Jordan blocks}

The final part of the problem concerns non-diagonal Jordan blocks. These arise for certain nongeneric plant structures and block the straightforward diagonalization based arguments used earlier. Those will only be enough to separate the problem into ones involving parallel real-valued positive 
Jordan blocks. To address it, it suffices to consider an $n$-dimensional square $A$ matrix that represents a single real Jordon block.

$$
A=\left[\begin{array}{ccccc}
\lambda & 1 & 0 & \cdots & 0 \\
0 & \lambda & 1 & \cdots & 0 \\
\vdots & 0 & \ddots & \ddots & 0 \\
0 & 0 & \cdots & 0 & \lambda
\end{array}\right]
$$

We will start with sufficiency:

Theorem 8.7 Assume that we have a noisy channel with access to noiseless feedback with an anytime rate region for some $\vec{\epsilon}>0$ that has $\left(\log _{2}\left(\vec{\lambda}_{\|}\right)+\vec{\epsilon}\right) \in \mathcal{R}_{\text {any }}\left(\eta \log _{2} \vec{\lambda}_{\|}+\vec{\epsilon}\right)$ where $\vec{\lambda}_{\|}$consists of the component-wise magnitudes of the $\lambda_{i}$.

Then we can stabilize the linear system with dynamics described by (27) with unstable eigenvalues $\lambda_{i}$, controllable $\left(A, B_{u}\right)$, observable $\left(A, C_{y}\right)$, by constructing an observer $\mathcal{O}$ and controller $\mathcal{C}$ for the unstable vector system that achieves $E\left[\left\|\vec{X}_{t}\right\|^{\eta}\right]<K$ for all sequences of bounded driving noise $\left\|\vec{W}_{t}\right\| \leq$ $\frac{\Omega}{2}$.

Proof: This is only a minor variation on theorem 8.3. The state components corresponding to diagonal Jordan blocks or stable dynamics are dealt with as before. For each non-diagonal Jordan block, the final state is also dealt with as before. For the other states, have to modify the construction in section 5.3 slightly. Since the dynamics has $X_{i+1}(t)$ in it as well as $W_{i}(t)$, we just add the $\Delta_{i+1}$ to the $\Omega$ while deciding the size of the box to keep the state in.

$$
\Omega_{i}=\Omega+\Delta_{i+1}
$$

Since that just makes the constant $\Omega$ effectively bigger, it does not change the asymptotic rate or the sense of reliability required.

It is clear that the same proof would generalize the finite-output-alphabet case of theorem 8.6 as well. The necessity part is where there is a true question. Does each eigenvalue add rate or is it only at the eigenvector level? The following theorem answers the question.

Theorem 8.8 Assume that for a given noisy channel, system dynamics described by (27) with eigenvalues $\lambda_{i}$ and $\eta>0$, that there exists an observer $\mathcal{O}$ and controller $\mathcal{C}$ for the unstable vector system that achieves $E\left[\left\|\vec{X}_{t}\right\|^{\eta}\right]<K$ for all sequences of bounded driving noise $\left\|\vec{W}_{t}\right\| \leq \frac{\Omega}{2}$.

Let $\left|\lambda_{i}\right|>1$ for $i=1 \ldots l$, and let $\vec{\lambda}$ be the l-dimensional vector consisting of only the exponentially unstable eigenvalues ${ }^{43}$ of $A$. Then for every $\overrightarrow{\epsilon_{1}}, \overrightarrow{\epsilon_{2}}>0$ we know that $\left(\log _{2} \overrightarrow{\lambda_{\|}}-\overrightarrow{\epsilon_{1}}\right) \in \mathcal{R}_{\text {any }}\left(\eta \log _{2} \overrightarrow{\lambda_{\|}}-\overrightarrow{\epsilon_{2}}\right)$ for the noisy channel considered with the encoder having access to noiseless feedback delayed by $1+\Theta\left(A, B_{u}, C_{y}\right)$.

This theorem tells us that each unstable eigenvalue, no matter whether it has its own eigenvector or not, induces a demand to reliably transport a bitstream. Before we get into the explicit proof of this necessity theorem, we will describe the decoding algorithm and prove a required lemma. Since the only new feature is the nondiagonal Jordan block, we concentrate just on that one block.

We encode $n$ parallel bitstreams at rates $\log _{2} \lambda>R=R_{1}=R_{2}=\cdots=R_{n}$ using the same bitencoder as was used in the previous part. At the decoder, notice that the last state is as before and

\footnotetext{
${ }^{43}$ If an eigenvalue has multiplicity, then it should appear in $\vec{\lambda}$ multiple times.
} 
only depends on its own bitstream. However, all the other states will have a mixture of bitstreams inside of them since the later states enter as inputs into the earlier states. As a result, the decoding algorithm given in section 4.3 .2 will not work as given on those states.

The decoding strategy in the non-diagonal Jordon block case ${ }^{44}$ will change to be successivedecoding in the style of decoding for the degraded broadcast channel in network information theory. [5] Explicitly, the procedure will go as follows:

1. Set $i=n$. Set $D_{j}(t)=-\tilde{X}_{j}(t)$ for all $j$.

2. Decode the bits on the $i$ th stream using the algorithm of section 4.3 .2 applied to $D_{i}(t)$.

3. Remove the impact of these bits from the $k$ th value of $D_{k}(t)$ for every $k<i$.

4. Decrement $i$ and goto step 2.

Notice that if all the bits decoded up to this point are correct, then at the time we come to decode the bits on the $i$ th stream using $D_{k}(t)$ as the input to the bit-extraction algorithm of section 4.3.2, the $D_{k}(t)$ will contain exactly what it would have contained had the $A$ matrix been diagonal. As such, the error probability calculations done earlier would apply. However, this successive decoding strategy has the possibility of propagating errors between streams and so we need to get a better handle on how this propagation can occur.

We first write the expression corresponding to equation (5) for the states $i<n$. By (7), we know $\left(2+\epsilon_{i}\right)=\lambda^{\frac{1}{R_{i}}}$ and so we can write:

$\check{X}_{i}(t)=\gamma \lambda^{t}\left[\left(\sum_{k=0}^{\lfloor R t\rfloor} \lambda^{-\frac{k}{R}} S_{i}(k)\right)+\left(\sum_{k=0}^{\lfloor R t\rfloor} \lambda^{-\frac{k}{R}} p_{1}(\lfloor R t\rfloor-k) S_{i-1}(k)\right)+\cdots+\left(\sum_{k=0}^{\lfloor R t\rfloor} \lambda^{-\frac{k}{R}} p_{n-i}(\lfloor R t\rfloor-k) S_{n}(k)\right)\right.$

where the $p_{k}$ represent polynomials. The key feature of polynomials we will use is that for every $\epsilon$, we can choose a $K_{i}$ so that $p_{k}(\tau) \leq K_{i} 2^{\epsilon \tau}$. We will bound the deviation possible if we make an error on all the bits after a certain point $t-d$.

Lemma 8.2 For every $\epsilon^{\prime}>0$, there exists a $K^{\prime}>0$ so that the maximum magnitude deviation of $D_{j}$ due directly to decoding errors in stream $i>j$ occurring for bits corresponding to times after $t-d$ is $K^{\prime} 2^{d\left(1+\epsilon^{\prime}\right) \log _{2} \lambda}$

Proof: The worst case is when every bit that could be wrong is wrong. Then the magnitude of the

\footnotetext{
${ }^{44}$ Actually, notice that all we use in this argument is that we have an upper-triangular block.
} 
deviation in $D_{j}$ due directly to decoding errors is given by:

$$
\begin{aligned}
\gamma \lambda^{t} \sum_{k=\lceil R(t-d)\rceil}^{\lfloor R t\rfloor} \lambda^{-\frac{k}{R}} p_{i-j}(\lfloor R t\rfloor-k) 2 & \leq 2 K \gamma \lambda^{t} \sum_{k=\lceil R(t-d)\rceil}^{\lfloor R t\rfloor} \lambda^{-\frac{k}{R}} 2^{\epsilon(\lfloor R t\rfloor-k)} \\
& \leq 2 K \gamma 2^{\left(R \epsilon+\log _{2} \lambda\right) t} \sum_{k=\lceil R(t-d)\rceil}^{\lfloor R t\rfloor} 2^{-k\left(\epsilon+\frac{\log _{2} \lambda}{R}\right)} \\
& \leq 2 K \gamma 2^{\left(R \epsilon+\log _{2} \lambda\right) t} 2^{-R(t-d)\left(\epsilon+\frac{\log _{2} \lambda}{R}\right)} \sum_{k=0}^{\infty} 2^{-k\left(\epsilon+\frac{\log _{2} \lambda}{R}\right)} \\
& =K^{\prime} 2^{\left(R t \epsilon+t \log _{2} \lambda\right)-(R t-R d)\left(\epsilon+\frac{\log _{2} \lambda}{R}\right)} \\
& =K^{\prime} 2^{d\left(\epsilon R+\log _{2} \lambda\right)} \\
& =K^{\prime} 2^{d\left(1+\frac{\epsilon R}{\log _{2} \lambda}\right) \log _{2} \lambda}
\end{aligned}
$$

Since $\epsilon$ was arbitrary, we can achieve this with any $\epsilon^{\prime}=\frac{\epsilon R}{\log _{2} \lambda}$.

Lemma 8.2 shows that the error propagation can only cause us a total deviation a little larger than $\lambda^{d}$. Since that is almost comparable to the error propagating up from our own stream's noise, we are ready to prove the theorem:

Proof of theorem 8.8: The goal of the $\overrightarrow{\epsilon_{2}}$ is to allow us to give an slightly lower sense of reliability to the early streams within a Jordon block. As in the sufficiency proof for theorem 8.7, the only new considerations in this proof occur with a single Jordon block and so we just concentrate on that case.

From (11), we know how much of a deviation in $D_{i}(t)$ we can tolerate and still have no error in decoding bits from before $d$ time steps ago. To get a bound on the probability of error, we will allocate that into $n-i+1$ equally-sized pieces. Each allocated margin will be of size $\frac{\gamma \epsilon_{1}}{(n-i+1)\left(1+\epsilon_{1}\right)} 2^{d \log _{2} \lambda}$. The first $n$ of them will correspond to an allowance for propagating errors. The final piece will correspond to what we allow from the controlled state.

Using lemma 8.2 and setting $d^{\prime}$ to the point in the past corresponding to the first error in the other stream, we can set the two terms equal to each other

$$
\begin{aligned}
\frac{\gamma \epsilon_{1}}{(n-i+1)\left(1+\epsilon_{1}\right)} 2^{d \log _{2} \lambda} & =K^{\prime} 2^{d^{\prime}\left(1+\epsilon^{\prime}\right) \log _{2} \lambda} \\
\frac{\gamma \epsilon_{1}}{K^{\prime}(n-i+1)\left(1+\epsilon_{1}\right)} 2^{d \log _{2} \lambda} & =2^{d^{\prime}\left(1+\epsilon^{\prime}\right) \log _{2} \lambda} \\
\frac{\log _{2}\left(\frac{\gamma \epsilon_{1}}{K^{\prime}(n-i+1)\left(1+\epsilon_{1}\right)}\right)}{\left(1+\epsilon^{\prime}\right) \log _{2} \lambda}+d \frac{1}{1+\epsilon^{\prime}} & =d^{\prime} \\
K^{\prime \prime}+d \frac{1}{1+\epsilon^{\prime}} & =d^{\prime}
\end{aligned}
$$

The key point to notice is that the tolerated delay $d^{\prime}$ on the other streams is a constant $K^{\prime \prime}$ plus a term that is almost equal to $d$.

As such, the probability of error on stream $i$ on bits at delay $d$ or earlier is bounded by

$$
\sum_{j=i+1}^{n} \mathcal{P}\left(\text { Stream } j \text { has an error at position } K^{\prime \prime}+d \frac{1}{1+\epsilon^{\prime}} \text { or earlier }\right)+P\left(\left|X_{i}(t)\right| \geq \frac{\gamma \epsilon_{1}}{(n-i+1)\left(1+\epsilon_{1}\right)} 2^{d \log _{2} \lambda}\right)
$$


We can immediately prove what we need by induction. The base case, $i=n$ is obvious since it is just it was in the scalar case by itself. Now assume that for every $j>i$, we have

$$
\mathcal{P}(\text { Stream } j \text { has an error at position } d \text { or earlier }) \leq K_{j}^{\prime \prime \prime} 2^{-d \frac{\eta \log _{2} \lambda}{\left(1+\epsilon^{\prime}\right)^{n-j}}}
$$

With the induction hypothesis and base case in hand, consider $i$ :

$\mathcal{P}($ Stream $i$ has an error at position $d$ or earlier $)$

$$
\begin{aligned}
& \leq \mathcal{P}\left(\left|X_{i}(t)\right| \geq \frac{\gamma \epsilon_{1}}{(n-i+1)\left(1+\epsilon_{1}\right)} 2^{d \log _{2} \lambda}\right)+\sum_{j=i+1}^{n} K_{j}^{\prime \prime \prime} 2^{-\left(K^{\prime \prime}+d \frac{1}{1+\epsilon^{\prime}}\right) \frac{\eta \log _{2} \lambda}{\left(1+\epsilon^{\prime}\right)^{n-j}}} \\
& =\mathcal{P}\left(\left|X_{i}(t)\right| \geq \frac{\gamma \epsilon_{1}}{(n-i+1)\left(1+\epsilon_{1}\right)} 2^{d \log _{2} \lambda}\right)+\sum_{j=i+1}^{n} K_{j}^{\prime \prime \prime} 2^{-K^{\prime \prime} \frac{\eta \log _{2} \lambda}{\left(1+\epsilon^{\prime}\right)^{n-j}}} 2^{-d \frac{\eta \log _{2} \lambda}{\left(1+\epsilon^{\prime}\right)^{n-j+1}}} \\
& \leq K^{\prime \prime \prime \prime \prime} 2^{-d \eta \log _{2} \lambda}+K_{i}^{\prime \prime \prime} 2^{-d \frac{\eta \log _{2} \lambda}{\left(1+\epsilon^{\prime}\right)^{n-i}}} \\
& \leq K_{i}^{\prime \prime \prime \prime \prime} 2^{-d \frac{\eta \log _{2} \lambda}{\left(1+\epsilon^{\prime}\right)^{n-i}}}
\end{aligned}
$$

where we used the induction hypothesis, the proof of theorem 4.3 and that the sum of exponentials is bounded by a constant times the slowest exponential. Since $\epsilon^{\prime}$ was arbitrary, we have proved the theorem since we can get as close as we want to $\alpha=\eta \log _{2} \lambda$ in anytime reliability.

It should be immediately clear that the arguments given in Part I on continuous-time models also apply to vector-valued state spaces. Standard results on sampling linear systems tell us that in the continuous-time case, the role of the magnitude of the unstable eigenvalues will be played by the real part of the unstable eigenvalues.

\section{Conclusions}

With theorems 8.8 and 8.7, we know that the problem of stabilization of a linear vector plant over a noisy channel is intimately connected to the problem of reliable sequential communication of parallel bitstreams in the anytime sense over a noisy channel where the encoder has access to noiseless feedback of the channel outputs. The anytime-capacity region of a channel with feedback is the key to understanding whether or not it is possible to stabilize an unstable linear system over that noisy channel. The two problems are related through three parameters. The primary role is played by the magnitudes of the unstable eigenvalues since their logs determine the required rates. The target moment $\eta$ multiplies these logs to give the required anytime reliabilities. Finally, the intrinsic delay $\Theta\left(A, B_{u}, C_{y}\right)$ tells us the noiseless feedback delay to use while evaluating the anytime reliabilities.

The primary limitation of the results so far is that they only cover the binary question of whether the plant is stabilizable in the $\eta$-moment sense or not. They do not address the issue of performance. In [23], we were able to cleanly approach the question of performance for the related scalar estimation problem. The linear systems techniques of part II apply directly to the estimation problem there and can generalize the result naturally to the vector case. For the estimation problem where the limit of large delays does not inherently degrade performance, it turns out that we require $l$ parallel bitstreams corresponding to each unstable eigenvalue, each of rate $>\log _{2}\left|\lambda_{i}\right|$, together with one residual bitstream that is used to boost performance in the end-to-end distortion sense. The unstable 
streams all require anytime reliability in the sense of theorem 8.8 while the residual stream just requires Shannon's traditional reliability. Since there are no control signals in the case of estimation, intrinsic delay plays no role there. In the control context, we already have the results from sequential rate distortion theory that give us a rate bound on the total bitrate required for a target level of performance.[33] However, there is no corresponding sufficiency result.

Because the results given here apply for general state-space models, we know that they apply to all equivalent linear models. In particular, they also apply to the case of systems modeled using ARMA models or with rational transfer functions of any finite order. Assuming that there is no pole/zero cancellation, such results can be proven using standard linear techniques establishing the equivalencies of such SISO models to the general state-space forms considered here. In those cases, the unstable eigenvalues of the state-space model will correspond to the unstable poles of the ARMA model. The intrinsic delay will correspond to the the number of leading zeros in the impulse response, i.e. the multiplicity of the pole at $z=0$.

\section{References}

[1] R. Bansal and T. Basar, "Simultaneous Design of Measurement and Control Strategies for Stochastic Systems with Feedback." Automatica, Volume 25, No. 5, pp 679-694, 1989.

[2] V. Borkar and S. Mitter, "LQG Control with Communication Constraints." In Communications, Computation, Control, and Signal Processing: a Tribute to Thomas Kailath. Norwell, MA, Kluwer Academic Publishers: 1997.

[3] M. V. Burnashev. "Data transmission over a discrete channel with feedback, random transmission time." Problemy Perdachi Informatsii, 12(4):10-30, 1976.

[4] M Costa, "Writing on dirty paper," IEEE Transactions on Information Theory, 29:439-441, May 1983.

[5] T. Cover and J. Thomas, Elements of Information Theory, John Wiley, New York, 1991.

[6] R. L. Dobrushin, "An asymptotic bound for the probability error of information transmission through a channel without memory using the feedback." Problemy Kibernetiki, Vol 8, pp 161-168, 1962 .

[7] N. Elia, "When Bode meets Shannon: control-oriented feedback communication schemes." IEEE Transactions on Automatic Control, pp. 1477- 1488, September 2004.

[8] N. Elia. "Stabilization of systems over analog memoryless channels." Submitted to Systems and Control Letters, May 2003

[9] R.G. Gallager, Information Theory and Reliable Communication. New York, NY: John Wiley and Sons, 1971.

[10] G. D. Forney, Jr., "Convolutional codes II. Maximum-likelihood decoding." Inform. and Control, vol. 25 pp. 222-266, 1974.

[11] G. D. Forney, Jr., "Convolutional codes III. Sequential decoding." Inform. and Control, vol. 25 pp. 267-297, 1974. 
[12] I. M. Jacobs and E. R. Berlekamp, "A lower bound to the distribution of computation for sequential decoding," IEEE Transactions on Information Theory, vol. 13, pp. 167-174, April 1967.

[13] A. J. Kramer, "Improving communication reliability by use of an intermittent feedback channel," IEEE Transactions on Information Theory, vol. IT-15, pp. 52 - 60, January 1969.

[14] G.N. Nair and R.J. Evans, "Communication-limited stabilization of linear systems," Proceedings of the 39th IEEE Conference on Decision and control vol 1, pp. 1005-1010, 2000.

[15] M. S. Pinsker, "Bounds of the Probability and of the Number of Correctable Errors for Nonblock Codes," Translation from Problemy Peredachi Informatsii, Vol. 3, No. 4, pp. 44-55, 1967.

[16] S. Rajagopalan, "A Coding Theorem for Distributed Computation." PhD Dissertation, University of California at Berkeley, 1994.

[17] A. Sahai, S. Tatikonda, S. Mitter, "Control of LQG Systems Under Communication Constraints." Proceedings of the 1999 American Control Conference, 1999.

[18] A. Sahai, "Evaluating Channels for Control: Capacity Reconsidered," Proceedings of the 2000 American Control Conference. ACC (IEEE Cat. No.00CH36334). American Autom. Control Council. Part vol.4, 2000, pp.2358-62 vol.4. Danvers, MA, USA

[19] A. Sahai, "Any-time Information Theory," PhD Dissertation, MIT February 2001.

[20] A. Sahai and S. K. Mitter, "A Fundamental Need for Differentiated 'Quality of Service' Over Communication Links: An Information Theoretic Approach.", Allerton Conference on Communication, Control, and Computing, 2000.

[21] A. Sahai and Q. Xu, "The Anytime Reliability of Constrained Packet Erasure Channels with Feedback," Allerton Conference on Communication, Control, and Computing 2004.

[22] A. Sahai and Q. Xu, "The Anytime Reliability of the AWGN+erasure channel with Feedback," Allerton Conference on Communication, Control, and Computing 2004.

[23] A. Sahai and S. K. Mitter, "Source coding and channel requirements for unstable processes." submitted to IEEE Transactions on Information Theory, November 2004.

[24] A. Sahai, T. Simsek, and P. Varaiya, "Upper-bounds to anytime reliability," In progress.

[25] J. P. M. Schalkwijk and T. Kailath, "A coding scheme for additive noise channels with feedback - I: No bandwidth constraint." IEEE Transactions on Information Theory, Vol 12, pp 172-182, April 1966.

[26] L. Schulman, "Coding for Interactive Communication." IEEE Transactions on Information Theory, Vol 42, pp. 1745-1756, November 1996.

[27] C. E. Shannon, "A Mathematical Theory of Communication." Bell System Technical Journal, vol. 27, pp. 379-423, 623-656, July and October 1948.

[28] C. Shannon, "The Zero Error Capacity of a Noisy Channel." IEEE Transactions on Information Theory, Vol 2, pp. S8-S19, September 1956. 
[29] C. E. Shannon, "Coding theorems for a discrete source with a fidelity criterion," IRE Nat. Conv. Rec., part 4, pp. 142-163, 1959.

[30] Tunc Simsek, "Anytime channel coding with feedback," Doctoral dissertation, Fall 2004.

[31] B. Sinopoli, L. Schenato, M. Franceschetti, K. Poolla, M. Jordan, S. Sastry, "Kalman filtering with intermittent observations," IEEE Transactions on Automatic Control, pp 1453- 1464, September 2004

[32] S. Tatikonda, A. Sahai, S. Mitter, "Control of LQG Systems Under Communication Constraints." Proceedings of the 37th IEEE Conference on Decision and Control, 1998.

[33] S. Tatikonda, A. Sahai, S. Mitter, "Stochastic Linear Control Over a Communication Channel," IEEE Transactions on Automatic Control, September 2004.

[34] S. Tatikonda, "Control Under Communication Constraints." PhD Dissertation, 2000.

[35] S. Tatikonda, S. Mitter, "Control Under Communication Constraints," accepted to IEEE Transactions on Automatic Control, February 2004.

[36] S. Tatikonda, S. Mitter, "Control Over Noisy Channels," accepted to IEEE Transactions on Automatic Control, February 2004.

[37] S. Vembu, S. Verdu, and Y. Steinberg, "The Source-Channel Separation Theorem Revisited." IEEE Transactions on Information Theory, 41(1):44-54, 1995.

[38] H. Witsenhausen, "Separation of Estimation and Control for Discrete Time Systems." Proceedings of the IEEE, Vol 59, No. 11, November 1971. 